ARTICLE

https://doi.org/10.1038/s41467-019-11291-w

\title{
Arabidopsis SWR1-associated protein methyl-CpG- binding domain 9 is required for histone H2A.Z deposition
}

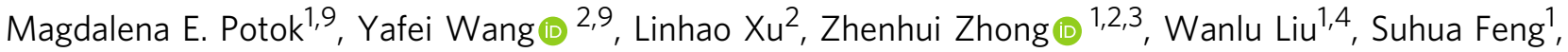 \\ Bilguudei Naranbaatar ${ }^{1}$, Shima Rayatpisheh (1) 5,8, Zonghua Wang ${ }^{3,6}$, James A. Wohlschlegel ${ }^{5}$, Israel Ausin (1) ${ }^{2}$ \& \\ Steven E. Jacobsen (1) ${ }^{1,7}$
}

Deposition of the histone variant H2A.Z by the SWI2/SNF2-Related 1 chromatin remodeling complex (SWR1-C) is important for gene regulation in eukaryotes, but the composition of the Arabidopsis SWR1-C has not been thoroughly characterized. Here, we aim to identify interacting partners of a conserved Arabidopsis SWR1 subunit ACTIN-RELATED PROTEIN 6 (ARP6). We isolate nine predicted components and identify additional interactors implicated in histone acetylation and chromatin biology. One of the interacting partners, methyl-CpGbinding domain 9 (MBD9), also strongly interacts with the Imitation SWItch (ISWI) chromatin remodeling complex. MBD9 is required for deposition of $\mathrm{H} 2 \mathrm{~A} . \mathrm{Z}$ at a distinct subset of ARP6-dependent loci. MBD9 is preferentially bound to nucleosome-depleted regions at the $5^{\prime}$ ends of genes containing high levels of activating histone marks. These data suggest that MBD9 is a SWR1-C interacting protein required for H2A.Z deposition at a subset of actively transcribing genes.

\footnotetext{
${ }^{1}$ Department of Molecular, Cell and Developmental Biology, University of California, Los Angeles, Los Angeles, CA 90095, USA. ${ }^{2}$ Basic Forestry and Proteomics Center, Fujian Agriculture and Forestry University, Fuzhou 350002, China. ${ }^{3}$ State Key Laboratory of Ecological Pest Control for Fujian and Taiwan Crops, Fujian Agriculture and Forestry University, Fuzhou 350002, China. ${ }^{4}$ Zhejiang University-University of Edinburgh Institute, Zhejiang University School of Medicine, Hangzhou 310058, China. ${ }^{5}$ Department of Biological Chemistry, David Geffen School of Medicine, University of California, Los Angeles, Los Angeles, CA 90095, USA. ${ }^{6}$ Institute of Oceanography, Minjiang University, Fuzhou 350108, China. ${ }^{7}$ Howard Hughes Medical Institute, University of California, Los Angeles, Los Angeles, CA 90095, USA. ${ }^{8}$ Present address: Genomic Institute of the Novartis Research Foundation, 10675 John Jay Hopkins Dr., San Diego, CA 92121, USA. ${ }^{9}$ These authors contributed equally: Magdalena E. Potok, Yafei Wang. Correspondence and requests for materials should be addressed to I.A. (email: israel.ausin@gmail.com) or to S.E.J. (email: jacobsen@ucla.edu)
} 
C hromatin dynamics plays a central role in regulating gene expression and has important implications in various cellular processes. The basic unit of chromatin is the nucleosome, which consists of 147 base-pairs (bp) of DNA wrapped around a histone octamer composed of two of each of the histones $\mathrm{H} 2 \mathrm{~A}, \mathrm{H} 2 \mathrm{~B}, \mathrm{H} 3$, and $\mathrm{H} 4$ (ref. ${ }^{1}$ ). Cells contain chromatin remodeling complexes, which modulate the packaging, spacing, and composition of nucleosomes ${ }^{2,3}$. The activity, targeting, and composition of remodeling complexes is tightly regulated to enable proper development of an organism ${ }^{2,3}$.

The most highly conserved histone variant in eukaryotes is $\mathrm{H} 2 \mathrm{~A}$. $\mathrm{Z}^{4-6}$. H2A.Z is found at many genes, most often around transcription start sites (TSS), with a particular preference for the +1 nucleosome $\mathrm{e}^{7-10}$. The relationship between H2A.Z accumulation and transcription is complex. When present in the active genes of some organisms, the levels of H2A.Z at the TSS positively correlate with gene expression, typically when found together with $\mathrm{H} 3 / \mathrm{H} 4$ acetylation, marks of active transcription ${ }^{10-13}$. H2A.Z is nonetheless absent at the most highly expressed genes ${ }^{7}$ and is also localized around the TSS of repressed genes that are poised for activation in yeast and animals $s^{9,14-16}$. Although the function of gene body methylation is unclear, one study in Arabidopsis suggests that a function of gene body DNA methylation may be to prevent H2A.Z deposition over housekeeping genes ${ }^{7,17}$, while another study found no such evidence ${ }^{18}$. However, it is clear that an anti-correlation between H2A.Z and DNA methylation exists across diverse systems ${ }^{16,19-21}$. In Arabidopsis, genes bearing high levels of gene body DNA methylation tend to be long and highly expressed, and show H2A.Z enrichment at their $5^{\prime}$-ends overlapping with activating histone modifications ${ }^{17,22-24}$. By contrast, enrichment of H2A.Z over gene bodies (including high H2A.Z levels at the TSS) is typically found in shorter, weakly expressed genes involved in environmental/stress responses that are marked with $\mathrm{H} 3 \mathrm{~K} 27 \mathrm{me} 3$ (refs. 17,22-25). Recently, levels of H3K27me3 were shown to be dependent on H2A.Z deposition in Arabidopsis ${ }^{23,26}$. Together, these studies demonstrate that H2A.Z can participate in both activation and repression of gene expression, likely in concert with other factors and chromatin marks.

H2A.Z is deposited by the SWI2/SNF2-Related 1 (SWR1) complex, which involves the replacement of H2A-H2B dimers by H2A.Z-H2B dimers ${ }^{27-29}$. In yeast, the SWR1 complex (SWR1-C) is composed of 14 subunits $^{30}$. In mammals, the structurally and functionally equivalent homolog of the yeast SWR1 complex, Snf2-Related CREBBP Activator Protein (SRCAP), is composed of 11 subunits, which are also conserved in yeast ${ }^{31-33}$. Based on homology, Arabidopsis contains one or several orthologs of the 11 conserved subunits from the yeast and human SWR1/SRCAP complexes, respectively ${ }^{34}$. Based on genetic and protein-protein interaction studies, several components have been validated as components of the Arabidopsis SWR1 complex, including PHOTOPERIOD-INDEPENDENT EARLY FLOWERING 1 (PIE1), the ATPase/scaffold subunit; ACTIN-RELATED PROTEIN 6 (ARP6); SERRATED LEAVES AND EARLY FLOWERING (SEF), the homolog of the yeast SWC6; and SWR1 complex subunit 2 (SWC2) ${ }^{35-37}$. A recent pull-down study of the SWC6 subunit identified additional subunits of the Arabidopsis SWR1 complex in yeast and validated their interaction with SWR1 complex subunit $4(\text { SWC4 })^{38}$. Mutations in the genes encoding the SWR1 subunits share various phenotypes, with null mutations in the core catalytic subunit PIE1 producing the most severe phenotypes, including reduced size, loss of apical dominance, reduced fertility, and early flowering $35-37,39-41$.

How the SWR1 complex is targeted across the genome remains unclear. Evidence suggests that H2A.Z localization around TSS in yeast is dependent on open chromatin and histone acetylation deposited by the Nucleosome acetyltransferase of H4 (NuA4) complex $^{8,42-46}$. The SWR1-C and NuA4 complexes share four common members: SWC4, YEAST ALL1-FUSED GENE FROM CHROMOSOME 9 (YAF9), ACTIN-RELATED PROTEIN 4 (ARP4), and ACTIN 1 (ACT1) 27-29,47-49. Homologs of these four proteins are present in the mammalian TIP60 complex, which possesses the dual activities of acetylating histones and depositing H2A.Z $Z^{31,50,51}$. The Arabidopsis genome also contains homologs for many of the yeast NuA4 subunits; however, a functional link between histone acetylation and H2A.Z deposition is unclear ${ }^{52}$. YAF9 proteins are required for both the proper expression of FLOWERING LOCUS C (FLC) and H2A.Z acetylation at its regulatory regions, but not for H2A.Z deposition ${ }^{53}$. Much more needs to be learned about the potential NuA4 complex in Arabidopsis, including its composition and genome-wide effects on H2A.Z deposition and modification. Although genome-wide localization of the SWR1 components is lacking in Arabidopsis, it has been reported that ARP6 and SWC4 directly bind their target locus FLOWERING LOCUS T $(F T)^{38}$; likewise, ARP6 and SWC6 bind directly to FLC ${ }^{35}$.

In this study, we use the conserved ARP6 subunit as bait to analyze the composition of the Arabidopsis SWR1 complex. We identify Arabidopsis homologs of most of the conserved SWR1 complex components in yeast, as well as several additional interacting partners: MBD9, four of the seven members of the ALFIN1-LIKE family (AL4, AL5, AL6, AL7), and two components of the yeast NuA4 and Arabidopsis SAGA histone acetyltransferase complexes, TRANSCRIPTION-ASSOCIATED PROTEIN 1A AND 1B (TRA1A and TRA1B). Our data are consistent with a related study where ARP6-interacting proteins were identified using ARP6-TAP-tag purification followed by mass spectrometry ${ }^{54}$. We focus on characterizing MBD9, a protein implicated in flowering time regulation ${ }^{55}$. Using biochemical, genetic, and transcriptome analyses, we show that MBD9 interacts with the SWR1 complex in Arabidopsis, and is required for H2A.Z deposition at a subset of ARP6-dependent genes. Furthermore, immunoprecipitation of MBD9 followed by mass spectrometry (IP-mass spec) reveals its association with the ISWI chromatin remodeling complex, suggesting additional functions for MBD9. Genome-wide ChIP-seq analyses of MBD9 show that it localizes to nucleosome-depleted regions of active genes but does not influence open chromatin itself. MBD9 is thus an interacting partner of the Arabidopsis SWR1 complex required for H2A.Z deposition at a subset of actively transcribed genes.

\section{Results}

MBD9 controls flowering time via its interaction with SWR1 complex. To identify components of the SWR1 complex in Arabidopsis, we used the conserved ARP6 subunit of the SWR1 complex as bait. We generated the epitope-tagged ARP6 lines pARP6:ARP6-3xFLAG and pARP6:ARP6-9xMYC. Both lines complemented arp6-1 mutants (Supplementary Fig. 1), indicating that epitope-tagged ARP6 is functional in vivo. To detect as many potential protein interactions as possible, we performed IP-mass spec in both FLAG- and MYC-tagged ARP6 lines using highly proliferating inflorescence tissue where levels of proteins are high. In all replicates (three biological replicates with two technical replicates each), we observed enrichment of the Arabidopsis homologs of most of the conserved yeast SWR1 complex components-ARP6, PIE1, RuvB-like protein 1 and 2 (RIN1, RIN2), ARP4, SWC4, SWC2, YAF9A, and SEF. This includes three of the four common subunits of the yeast NuA4 complex, ARP4, SWC4, and YAF9A (Table 1). Our experiment confirms previous wellcharacterized in vivo and in vitro interactions of ARP6 with PIE1 and SEF ${ }^{36-38}$. We additionally identified two homologs of the yeast Tra1 proteins, subunits of the yeast NuA4 complex, TRA1A 
Table 1 IP-mass spectrometry of ARP6

\begin{tabular}{|c|c|c|c|c|c|c|c|c|c|c|c|c|c|}
\hline \multirow[b]{2}{*}{ Protein } & \multirow[b]{2}{*}{ Annotation } & \multicolumn{4}{|c|}{ ARP6-9xMYC } & \multicolumn{4}{|c|}{ Wild type } & \multicolumn{2}{|c|}{ ARP6-3xFLAG } & \multicolumn{2}{|c|}{ Wild type } \\
\hline & & $\mathbf{R 1}$ & R2 & R3 & R4 & R1 & $\mathbf{R 2}$ & R3 & R4 & R1 & $\mathbf{R 2}$ & $\mathbf{R 1}$ & $\mathbf{R 2}$ \\
\hline AT3G33520 & ARP6 & 123 & 119 & 138 & 123 & 0 & 0 & 0 & 0 & 573 & 501 & 0 & 0 \\
\hline AT3G12810 & PIE1 & 175 & 208 & 206 & 202 & 0 & 0 & 0 & 0 & 76 & 82 & 3 & 0 \\
\hline AT5G22330 & RIN1 & 176 & 189 & 224 & 196 & 3 & 4 & 0 & 0 & 62 & 56 & 5 & 7 \\
\hline AT5G67630 & RIN2 & 115 & 136 & 175 & 146 & 3 & 2 & 2 & 0 & 51 & 41 & 7 & 5 \\
\hline AT1G18450 & ARP4 & 75 & 76 & 64 & 56 & 8 & 3 & 5 & 2 & 35 & 37 & 9 & 10 \\
\hline AT2G47210 & SWC4 & 45 & 46 & 33 & 39 & 0 & 0 & 0 & 0 & 30 & 25 & 0 & 0 \\
\hline AT2G36740 & SWC2 & 37 & 32 & 35 & 34 & 0 & 0 & 0 & 0 & 14 & 11 & 0 & 0 \\
\hline AT5G45600 & YAF9A & 25 & 19 & 20 & 21 & 0 & 0 & 0 & 0 & 13 & 12 & 0 & 0 \\
\hline AT5G37055 & SEF & 11 & 9 & 9 & 8 & 0 & 0 & 0 & 0 & 9 & 8 & 0 & 0 \\
\hline AT3G01460 & MBD9 & 67 & 71 & 97 & 108 & 0 & 0 & 0 & 0 & 10 & 11 & 0 & 0 \\
\hline AT2G17930 & TRA1A & 84 & 94 & 117 & 117 & 0 & 0 & 0 & 0 & 31 & 25 & 0 & 0 \\
\hline AT4G36080 & TRA1B & 72 & 78 & 91 & 91 & 0 & 0 & 0 & 0 & 19 & 17 & 0 & 0 \\
\hline AT5G26210 & AL4 & 2 & 3 & 2 & 2 & 0 & 0 & 0 & 0 & 0 & 0 & 0 & 0 \\
\hline AT5G20510 & AL5 & 12 & 16 & 15 & 14 & 0 & 0 & 0 & 0 & 3 & 4 & 0 & 0 \\
\hline AT2G02470 & AL6 & 5 & 6 & 4 & 5 & 0 & 0 & 0 & 0 & 2 & 0 & 0 & 0 \\
\hline AT1G14510 & AL7 & 6 & 3 & 5 & 6 & 0 & 0 & 0 & 0 & 0 & 0 & 0 & 0 \\
\hline
\end{tabular}

Spectral counts for ARP6-9xMyc and ARP6-3xFlag lines representing enriched genes over wild type. Source data are provided as Source Data file

and TRA1B. We also observed consistent enrichment for several interacting partners previously implicated in chromatin biology, MBD9 (refs. ${ }^{55,56}$ ) and AL4, AL5, AL6, and AL7, which recognize di- and trimethyl H3K4 histone marks ${ }^{57}$. The interaction between ARP6 and MBD9 was further validated by coimmunoprecipitation (co-IP) in Arabidopsis flowers using an antibody raised against MBD9 (Fig. 1a). We then generated epitope-tagged MBD9 lines, $p M B D 9: M B D 9-3 x F L A G$ and $p M B D 9$ : MBD9-9xMYC. Both lines complemented mbd9-3 mutants (Supplementary Fig. 2), indicating that the epitope-tagged MBD9 is functional in vivo. We performed IP-mass spec using floral tissue from both FLAG- and MYC-tagged MBD9 lines. In both experiments we observed enrichment of the SWR1 complex components PIE1, RIN1, ARP4, RIN2, SWC2, ARP6, SWC4, YAF9A, and SEF (Table 2). Interestingly, we also identified TRA1A and TRA1B, components of the NuA4 complex, as common interacting partners between ARP6 and MBD9. Additionally, in our MBD9 IP-mass spec we detected high enrichment of ISWI chromatin remodeling complex members that were not preferentially enriched among the ARP6-associated proteins.

To explore the genetic interaction between MBD9 and ARP6, we used the T-DNA mutants arp6-1 and mbd9-3 (refs. ${ }^{35,39,40,55,56}$ ). Both single mutants displayed an early flowering time phenotype (Fig. 1c) as described previously $39,40,55$. The $m b d 9-3$ phenotype was less severe than that of arp6-1;mbd9-3 plants were larger, with a leaf morphology that more closely resembled wild-type plants (Fig. 1b). The mbd9-3 mutant plants also flowered slightly later than the arp6-1 mutants under both long-day (LD) and short-day (SD) conditions (Fig. 1c). The arp61 mbd9-3 double mutants had a more severe developmental phenotype than either of the single mutants, which resembled the strong phenotype of pie1-2, a mutant affecting the catalytic subunit of the SWR1 complex (Fig. 1b) $35,37,58$. The stronger phenotype of the arp6-1 mbd9-3 double mutant was also observed in a related study by Sijacic et al. ${ }^{54}$. Despite the severe developmental phenotype, the double mutant had a similar flowering time to arp6-1 under both LD and SD conditions; however, the time until anthesis (floral opening) was slightly shorter under SD growth conditions in the double mutant than the arp6-1 mutant (Fig. 1c), most likely due to morphological defects present in the arp6-1 mbd9-3 double mutant. These data suggest that, regarding flowering time, ARP6 is epistatic to MBD9 in both $\mathrm{LD}$ and $\mathrm{SD}$ conditions. Expression analysis of key flowering time genes, FLC and MADS AFFECTING FLOWERING 4 AND 5 (MAF4 and MAF5), indicated that both the single mutants and the double mutant had a similar reduction of expression of these genes (Fig. 1d). Together, these results indicate that MBD9 and ARP6 act together in the same genetic pathway controlling flowering time as part of the SWR1 complex.

MBD9 is required for the deposition of H2A.Z. To investigate the role of MBD9 in H2A.Z deposition, we analyzed H2A.Z levels in Arabidopsis seedlings before the major developmental transition to flowering in order to minimize secondary effects in mutants. We performed western blot on total extracts, and observed the levels to decline in the mutants: wild type $>m b d 9-3$ >arp6-1 > arp6-1 mbd9-3 (Fig. 2a). To investigate the impact of H2A.Z loss on chromatin, we performed two replicates of H2A.Z ChIP-seq in wild type, $m b d 9-3$, arp6-1, and arp6-1 mbd9-3, with H3 ChIP-seq as a control. Pearson correlation indicated a considerable overlap between the ChIP-seq replicates (Supplementary Fig. 3a). As with H2A.Z protein levels, we detected a sequential decrease in H2A.Z signal over H2A.Z peaks in wild type and over protein-coding genes as follows: wild type $>m b d 9$ $3>\operatorname{arp6}-1>\operatorname{arp6}-1$ mbd9-3 (Fig. 2b). By contrast, $\mathrm{H} 3$ levels were unchanged over these regions (Supplementary Fig. 3b). Using the MACS2 peak caller for each replicate $(q$ value $<0.01)$, we defined common H2A.Z differential regions for each genotype (Supplementary Methods). We surveyed the gene annotations in each differential region and identified genes that lost H2A.Z in the mutants compared to wild type: 2594 for $m b d 9-3,12,222$ for arp6-1, and 15,226 for arp6-1 mbd9-3. GO term enrichment for the three groups of genes yielded similar results, including postembryonic development and protein modification, indicating that broad classes of genes were impacted by the loss of H2A.Z in MBD9 and ARP6 mutants (Supplementary Fig. 3c).

Comparison of the H2A.Z-depleted genes in each mutant revealed a high degree of overlap (Fig. 2c). A metaplot of H2A.Z occupancy over the different groups of genes revealed that: (1) although mbd9-3 mutants had fewer genes that lost H2A.Z than arp6-1, the depletion of H2A.Z over $m b d 9-3$-dependent loci was nearly as great as in $\operatorname{arp6}-1$ (Fig. 2d); 2), genes that lost H2A.Z in arp6-1 and arp6-1 mbd9-3 behaved similarly, with H2A.Z enrichment following the order: wild type $>\operatorname{mbd9-3}>\operatorname{arp6}-1>$ arp6-1 mbd9-3 (Fig. 2e, f). We also defined 782 genes that required both MBD9 and ARP6 for H2A.Z deposition (arp6-1 

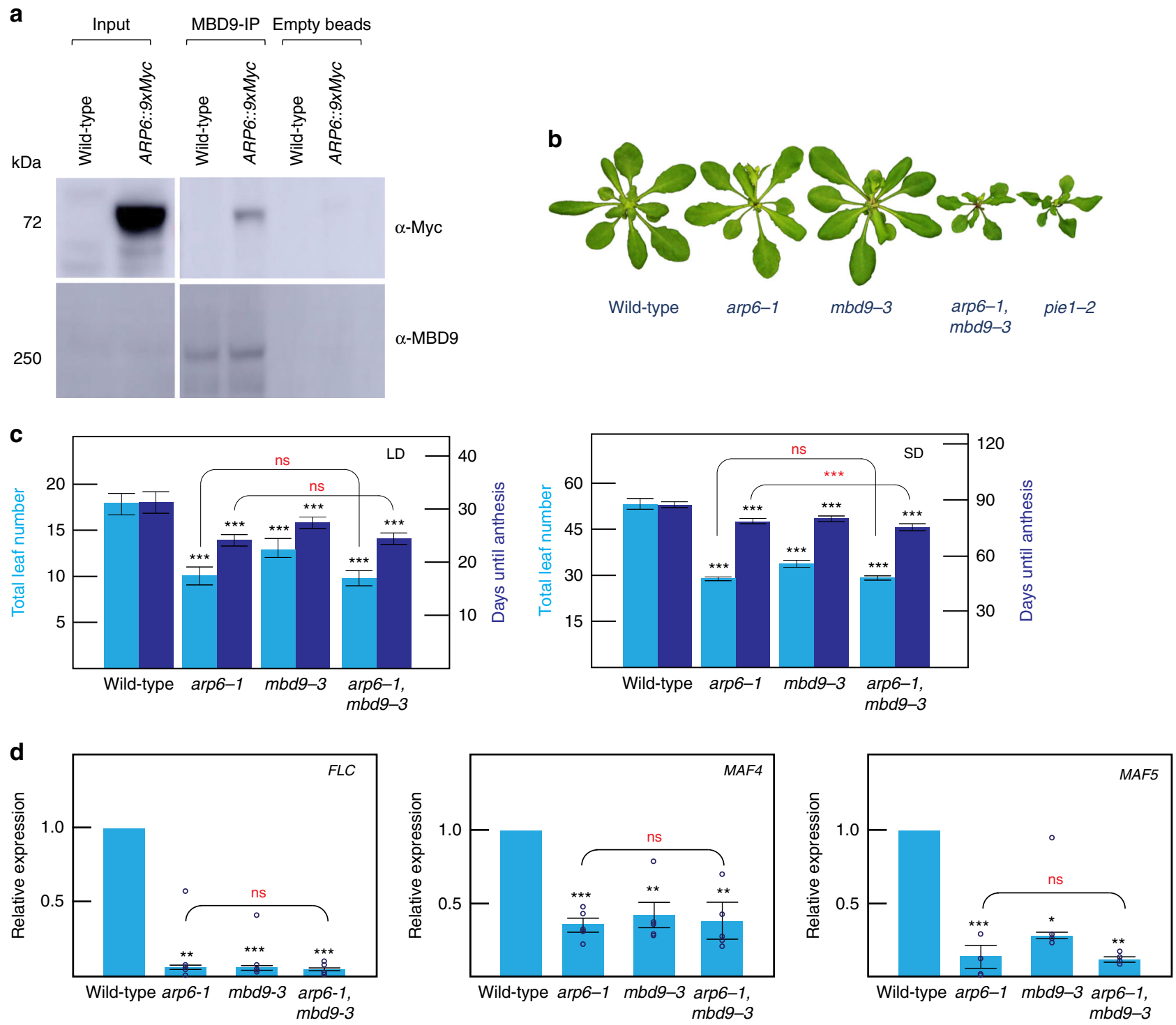

Fig. 1 ARP6 and MBD9 interaction and phenotype of the mutants. a Pull-down and co-immunoprecipitation assay. Empty bead lanes serve as negative controls. For each western blot, the antibody used is indicated to the right. b Phenotype of arp6-1, mbd9-3, arp6-1 mbd9-3, and pie1-2 mutants. Plants were grown for 4 weeks (b) or 5 weeks (c) under long-day (LD) conditions ( $16 \mathrm{~h}$ light, $8 \mathrm{~h}$ dark) and short-day (SD) conditions ( $8 \mathrm{~h}$ light, $16 \mathrm{~h}$ dark). Flowering time expressed as the number of days from germination to anthesis as well as the total number of leaves produced by wild type, arp6-1, mbd9-3, and arp6-1 mbd9-3 under the same conditions are shown. Averages from 16 plants \pm standard deviations are shown. Paired two-tailed Student's $t$-test was used to determine significance between wild type and mutants (black asterisks) or between mutants (red asterisks); ns $p$ value $>0.05,{ }^{\star} p$ value $\leq 0.05$, ${ }^{\star \star} p$ value $\leq$ 0.01, ${ }^{\star \star \star} p$ value $\leq 0.001$. d Relative expression levels of FLC, MAF4, and MAF5 in wild type, arp6-1, mbd9-3, and arp6-1 mbd9-3. UBQ10 was used as an internal control for expression analyses. Average expression levels from three independent replicates \pm standard deviations are shown. Paired two-tailed Student's $t$-test was used to determine significance between wild type and mutants (black asterisks) or between mutants (red asterisks); ns $p$ value $>0.05$, ${ }^{\star} p$ value $\leq 0.05,{ }^{\star \star} p$ value $\leq 0.01,{ }^{\star \star \star} p$ value $\leq 0.001$. Source data for Figs. 1a, $1 c$, and $1 d$ are provided as Source Data file

mbd9-3 mutant vs arp6-1, $q$ value <0.01). These genes were enriched for cell cycle progression functions (Supplementary Fig. 3c). The H2A.Z profiles over this set of genes (Fig. 2g) support the observation that these regions were dependent on both MBD9 and ARP6 for H2A.Z deposition. In Arabidopsis, housekeeping genes typically contain H2A.Z exclusively over the +1 nucleosome and lowly expressed/responsive genes contain H2A.Z over the entire gene bodies, with highest levels of H2A.Z at the TSS ${ }^{17,23,24}$. To investigate which types of genes are most impacted by the loss of H2A.Z in our mutants, we divided genes (into nine classes) according to the levels of H2A.Z in wild type over gene bodies (low, medium, and high) and TSS (low, medium, and high) as described in ref. ${ }^{17}$ (Supplementary Fig. 4, Supplementary Methods). We intersected these classes of gene with genes that lost H2A.Z in our mutants and plotted their distribution (Fig. 2h). This revealed that loss of H2A.Z in the mbd9-3 mutant tends to occur over genes possessing high levels of H2A.Z over the TSS and low levels of H2A.Z over gene bodies (HL class-High TSS, Low gene body), whereas loss of H2A.Z in the arp6-1 mutant tends to occur over genes containing high levels of H2A.Z over gene bodies ( $\mathrm{LH}, \mathrm{MH}$, and $\mathrm{HH}$ classes) (Fig. 2h).

MBD9/ARP6 partial redundancy in gene expression control. To investigate the relationship between H2A.Z loss and transcription, we performed RNA-seq in seedlings in wild type, $m b d 9-3$, arp6-1, and arp6-1 mbd9-3. We defined differentially 
Table 2 IP-mass spectrometry of MBD9

\begin{tabular}{llllll} 
Protein & Annotation & MBD9-9xMYC & MBD9-3xFLAG & Wild-type-9xMYC & Wild-type-3xFLAG \\
\hline AT3G01460 & MBD9 & 355 & 607 & 0 & 0 \\
AT3G12810 & PIE1 & 75 & 49 & 0 & 0 \\
AT5G22330 & RIN1 & 33 & 26 & 0 & 10 \\
AT1G18450 & ARP4 & 31 & 24 & 0 & 9 \\
AT5G67630 & RIN2 & 29 & 22 & 0 & 13 \\
AT2G36740 & SWC2 & 17 & 0 & 0 & 0 \\
AT3G33520 & ARP6 & 16 & 5 & 0 & 0 \\
AT2G47210 & SWC4 & 12 & 9 & 0 & 0 \\
AT5G45600 & YAF9A & 5 & 6 & 0 & 0 \\
AT5G37055 & SEF & 2 & 0 & 0 & 0 \\
AT2G17930 & TRA1A & 128 & 76 & 0 & 0 \\
AT4G36080 & TRA1B & 120 & 74 & 0 & 0 \\
AT2G02470 & AL6 & 2 & 0 & 0 & 0 \\
AT5G18620 & CHR17 & 122 & 190 & 0 \\
AT3G0640 & CHR11 & 118 & 177 & 0 & 0 \\
\hline
\end{tabular}

Spectral counts for MBD9-9xMyc and MBD9-3xFlag lines representing enriched genes over wild type. Source data are provided as Source Data file

expressed genes (fold change $>2$, FDR $<0.05$, Supplementary Methods). We detected 277 misregulated genes in $m b d 9-3,2042$ in arp6-1, and 1620 in arp6-1 mbd9-3. Among the downregulated genes we detected FLC, MAF4, and MAF5 as targets of both MBD9 and ARP6, confirming our RT-qPCR results (Supplementary Fig. 5a). Importantly, the expression of genes encoding the predicted subunits of the SWR1 complex as well as H2A.Z genes were largely unaffected in the mutants (Supplementary Fig. 5b). We examined down-regulated and up-regulated genes (Fig. 3) and observed a high degree of overlap between the arp6-1 and arp6-1 mbd9-3 mutants, with 584 common upregulated and 616 common down-regulated genes (Fig. 3a, b). These overlapping genes showed the same trend in expression change in mbd9-3 mutants, but to a lesser degree that did not meet our significance cutoffs (Fig. $3 c$, d). We also defined genes specifically up- and down-regulated in either $m b d 9-3$ or arp6-1, implying independent functions at a subset of loci (Fig. 3a-d) as well as a set of 218 up-regulated genes that were dependent on both ARP6 and MBD9 for expression (Fig. 3a, c), suggesting a redundant role of MBD9 and ARP6 at this set of genes. By contrast, the unique down-regulated genes $(n=170)$ did not show a significant change between arp6-1 and arp6-1 mbd9-3 (Fig. 3b, d). GO term analysis indicated that up-regulated genes in mbd9-3 mutants are involved in post-embryonic development and response to stress, whereas for arp6-1 and arp6-1 mbd9-3 upregulated genes are involved in lipid localization, biosynthetic processes, and response to drug (Supplementary Fig. 5c). By contrast, down-regulated genes are involved in response to stress, stimulus, and defense in all mutants (Supplementary Fig. 5c).

We compared differentially expressed genes in relation to $\mathrm{H} 2 \mathrm{~A}$. $\mathrm{Z}$ changes in the mutants. First, to obtain general trends, we generated boxplots of expression and profiles of $\mathrm{H} 2 \mathrm{~A} . \mathrm{Z}$ in the nine classes of genes divided according to their H2A.Z occupancy (Supplementary Fig. 4) for wild type, $m b d 9-3$, arp6-1, and arp6-1 mbd9-3 (Supplementary Fig. 6a, b). One general trend stood out, genes containing high-medium H2A.Z levels over gene bodies and the highest H2A.Z levels over TSS showed increased expression upon loss of H2A.Z in arp6-1, which was even more pronounced in the arp6-1 mbd9-3 mutant (Supplementary Fig. 6a, b). Only a weak loss of H2A.Z was observed in the mbd9-3 mutant over these classes of genes where expression was not significantly increased (Supplementary Fig. 6a, b). Interestingly, looking at a typical H2A.Z profile of housekeeping genes (low H2A.Z at gene body with high levels of H2A.Z at TSS), H2A.Z deposition depended on both ARP6 and MBD9; however, this loss did not cause major transcriptional changes even in arp6-1 mbd9-3 where H2A.Z levels were nearly depleted (Supplementary Fig. 6a, b). This perhaps is not surprising since genes that typically change in expression (become up-regulated) upon H2A. $\mathrm{Z}$ misregulation are weakly expressed/environmentally responsive genes that contain highest levels of H2A.Z over the gene body and the TSS $17,23-25,59,60$, most likely reflecting changes in chromatin accessibility $^{23,25}$. We next profiled H2A.Z changes in the significantly up- and down-regulated genes in our RNA-seq datasets (Fig. 3e). Both up- and down-regulated genes lost H2A.Z in arp6-1 and arp6-1 mbd9-3 mutants (Supplementary Fig. 5d). In agreement with the observed general trends, the up-regulated genes tend to belong to gene classes containing high levels of $\mathrm{H} 2 \mathrm{~A} . \mathrm{Z}$ over the gene body ( $\mathrm{LH}, \mathrm{MH}, \mathrm{HH}$ classes). More specifically, loss of $\mathrm{H} 2 \mathrm{~A} . \mathrm{Z}$ in $m b d 9-3$ was observed most over genes up-regulated in $m b d 9-3(n=84$ and $n=33)$ and those specifically up-regulated in arp6-1 $m b d 9-3(n=218)$ (Supplementary Fig. 5d), suggesting that MBD9-dependent H2A.Z deposition may be directly involved in repression of this subset of genes. $\operatorname{arp6}-1$ dependent up-regulated genes $(n=252$ and $n=$ 500 ) had minimal if any change in H2A.Z levels in mbd9-3 (Supplementary Fig. 5d). The down-regulated genes did not tend to belong to any particular class of H2A.Z-containing genes (Fig. 3e). Except for few well-studied genes (MADS-box proteins), the down-regulated genes in the SWR1-C mutants (including MBD9) most likely reflect indirect effects related to H2A.Z loss perhaps due to misregulated transcription factors in our mutants (Supplementary Fig. 7). Altogether, these data suggest that (1) mbd9-3 has a much weaker effect on transcription than arp6-1; (2) genes misregulated in the $m b d 9-3$ background are a subset of genes misregulated in the arp6-1 background; (3) MBD9 and ARP6 also cooperate to control the expression of a subset of upregulated genes; and (4) although loss of H2A.Z is observed genome-wide in arp6-1, and is even more pronounced in arp6-1 $m b d 9-3$, genes that change in expression upon the loss of H2A.Z are generally those that contain high levels of H2A.Z at TSS and high-medium levels of H2A.Z over the gene body.

MBD9 occupies nucleosome-depleted regions near active genes. To determine the localization of MBD9 in chromatin, we performed MBD9 ChIP-seq on our complementing pMBD9: MBD9-3xFLAG T4 transgenic line, with wild type as a control (Supplementary Fig. 2). Using MACS2 ( $q$ value $<0.01$ ), we defined 10,348 MBD9-occupied peaks, corresponding to 5772 genes 


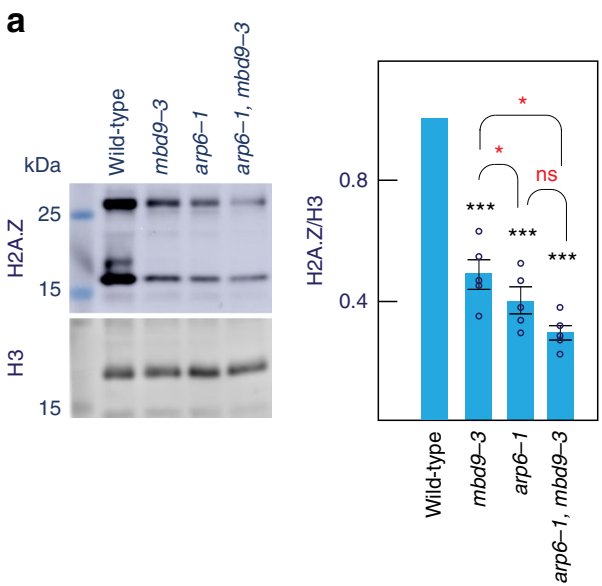

b

C

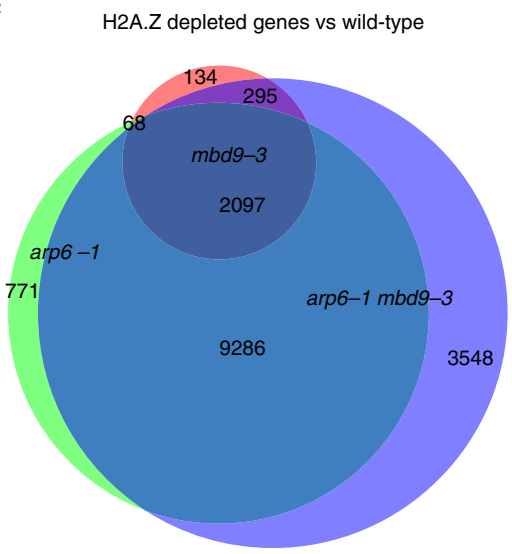

d
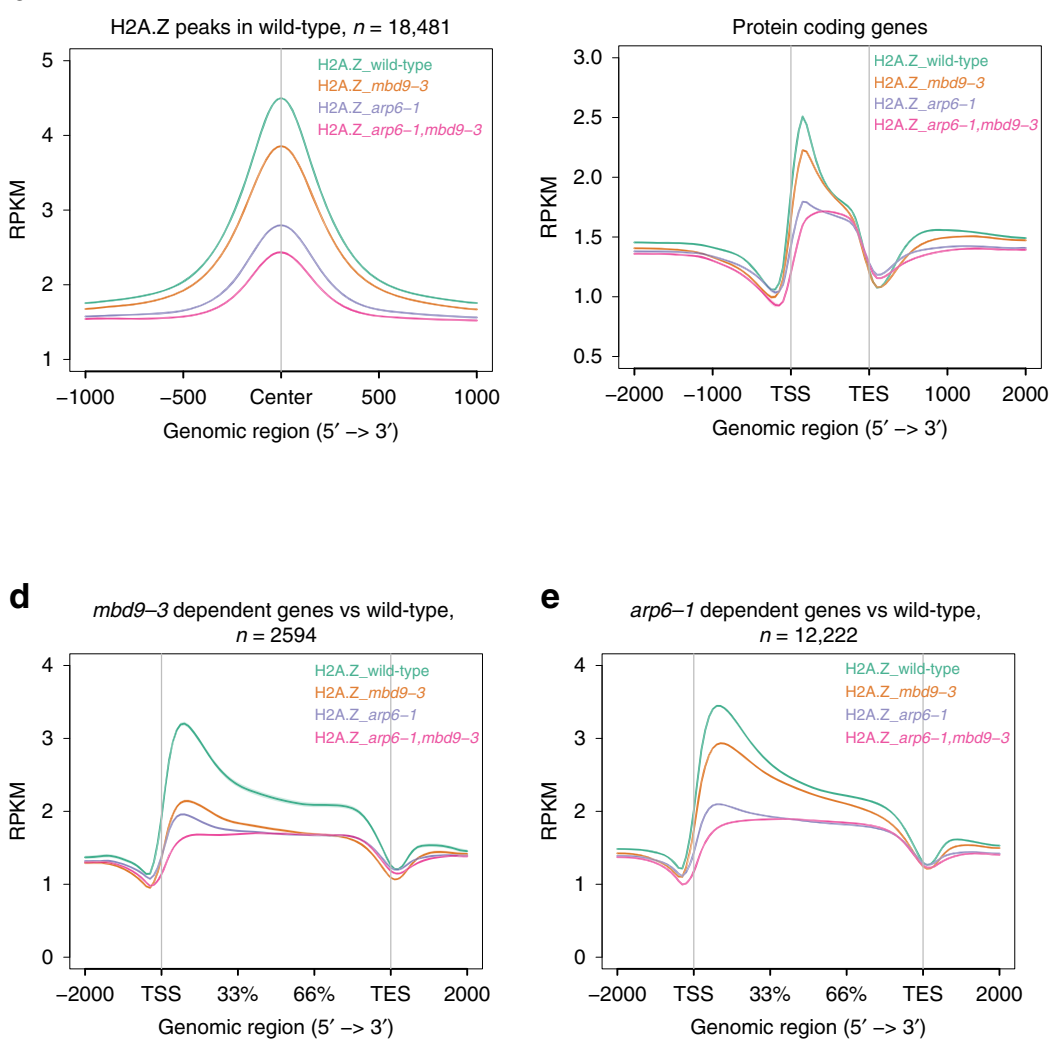

e

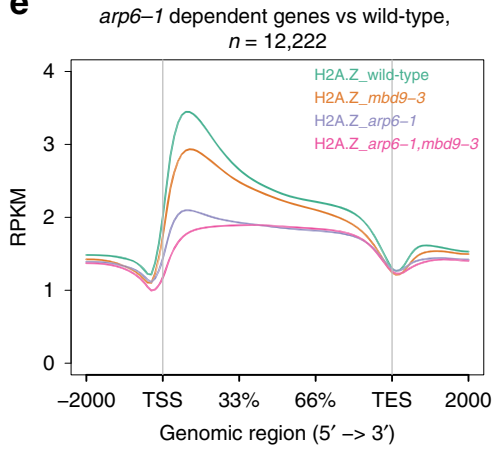

f arp6-1 mbd9-3 dependent vs wild-type,

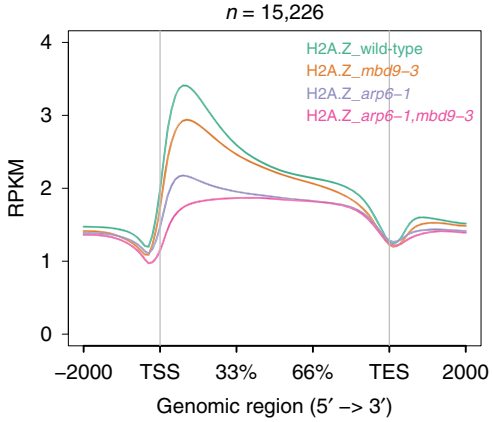

g

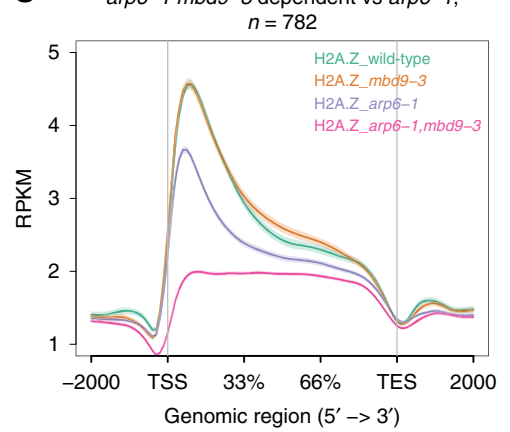

h

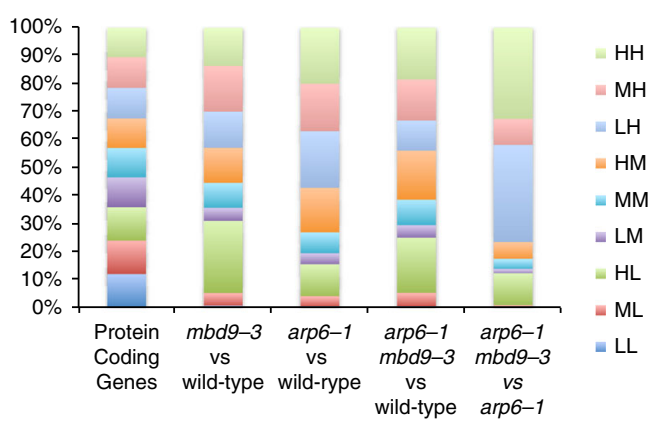

Fig. 2 Characterization of H2A.Z in wild type and mutant chromatin. a Western blot and quantification for H2A.Z and H3 levels in total extracts from wild type, $m b d 9-3$, arp6-1, and arp6-1 mbd9-3. The upper band in the H2A.Z blot likely represents a modified form of H2A.Z. Only the lower band was used in the quantification. Average levels of three independent westerns \pm standard deviations are shown. Paired two-tailed Student's $t$-test was used to determine significance between wild type and mutants (black asterisks) or between mutants (red asterisks); ns $p$ value $>0.05,{ }^{*} p$ value $\leq 0.05$, ${ }^{\star *} p$ value $\leq 0.01,{ }^{\star \star *} p$ value $\leq=0.001$. $\mathbf{b}$ Distribution of normalized H2A.Z ChIP-seq signal as read count kbp ${ }^{-1}$ million $^{-1}$ mapped reads (RPKM) from merged replicates over H2A.Z common peaks in wild type and protein-coding genes. c Overlap of H2A.Z-depleted genes for mbd9-3, arp6-1, and arp6-1 mbd9-3 mutants. d-g Distribution of normalized H2A.Z ChIP-seq signal (RPKM) from merged replicates over H2A.Z-enriched genes for indicated mutants. $\mathbf{h}$ Intersection of $\mathrm{H} 2 \mathrm{~A}$.Z-depleted genes from $\mathbf{d}$ to $\mathbf{g}$ with nine classes of genes characterized by their distribution of H2A.Z in wild type at TSS and gene body. Labels $\mathrm{H}-$ high, $\mathrm{M}-$ medium, L-low refer to levels of H2A.Z at TSS (first letter) and gene body (second letter). Source data for Figs. 2a and 2c are provided as Source Data file

having a peak within $1 \mathrm{~kb}$ upstream of the TSS (Supplementary Methods). The distribution of all MBD9 peaks showed a preference for promoter and intergenic regions (68\%) compared to a control set of peaks randomly selected from the genome based on length and number (Fig. 4a). Although MBD9 contains a methylCpG-binding domain, it is predicted not to bind methylated DNA since it lacks key conserved residues required for $\mathrm{mCpG}$ binding $^{61}$. Consistently, the level of methylation over MBD9 peaks in wild-type plants was lower in all cytosine contexts than over a control set of peaks (Fig. 4b).

We plotted MBD9 peaks alongside open chromatin regions, as determined by ATAC-seq, given MBD9's clear preference for promoter regions. This revealed a significant overlap between MBD9 peaks and open chromatin regions (Fig. 4c). At these regions, the open chromatin was largely unaffected in mbd9-3 (Fig. 4c). Metaplots of MBD9 ChIP-seq signal over 
a
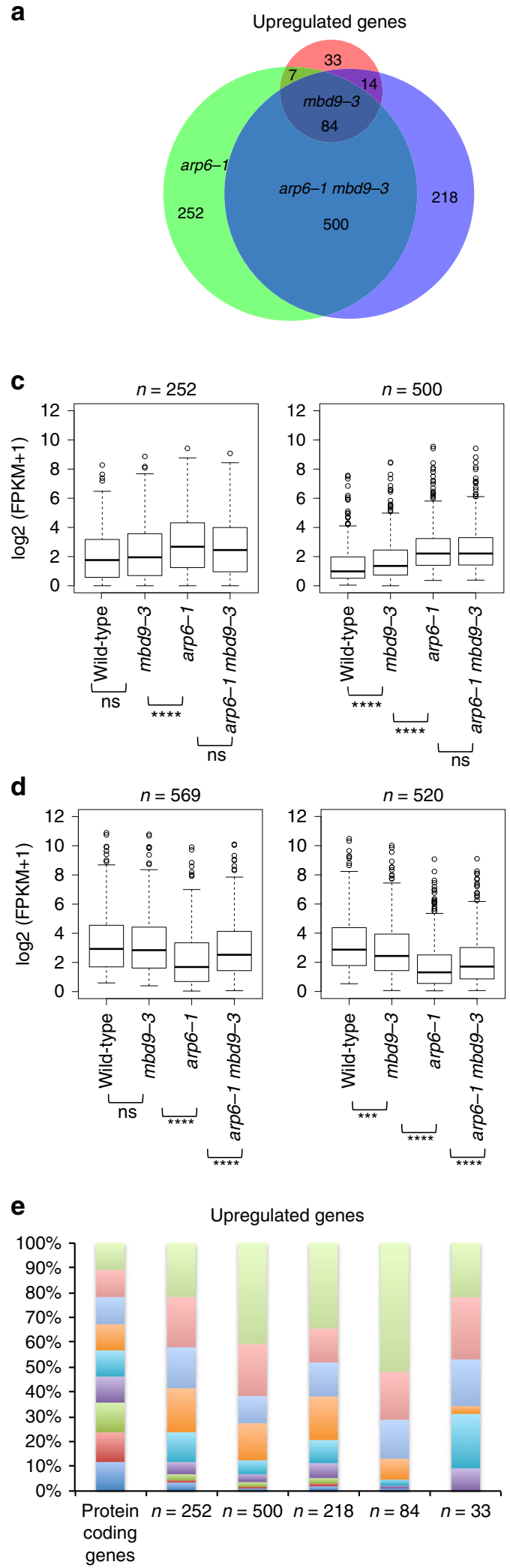

b

Downregulated genes

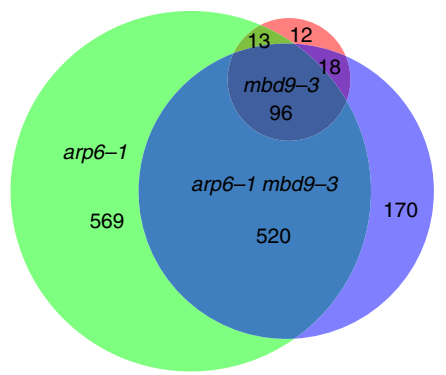

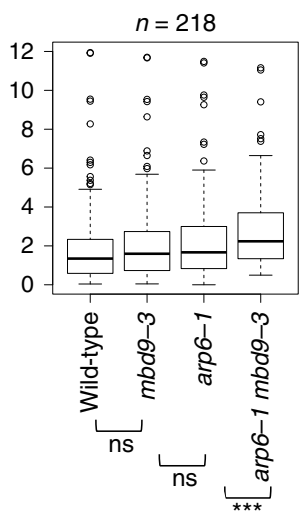
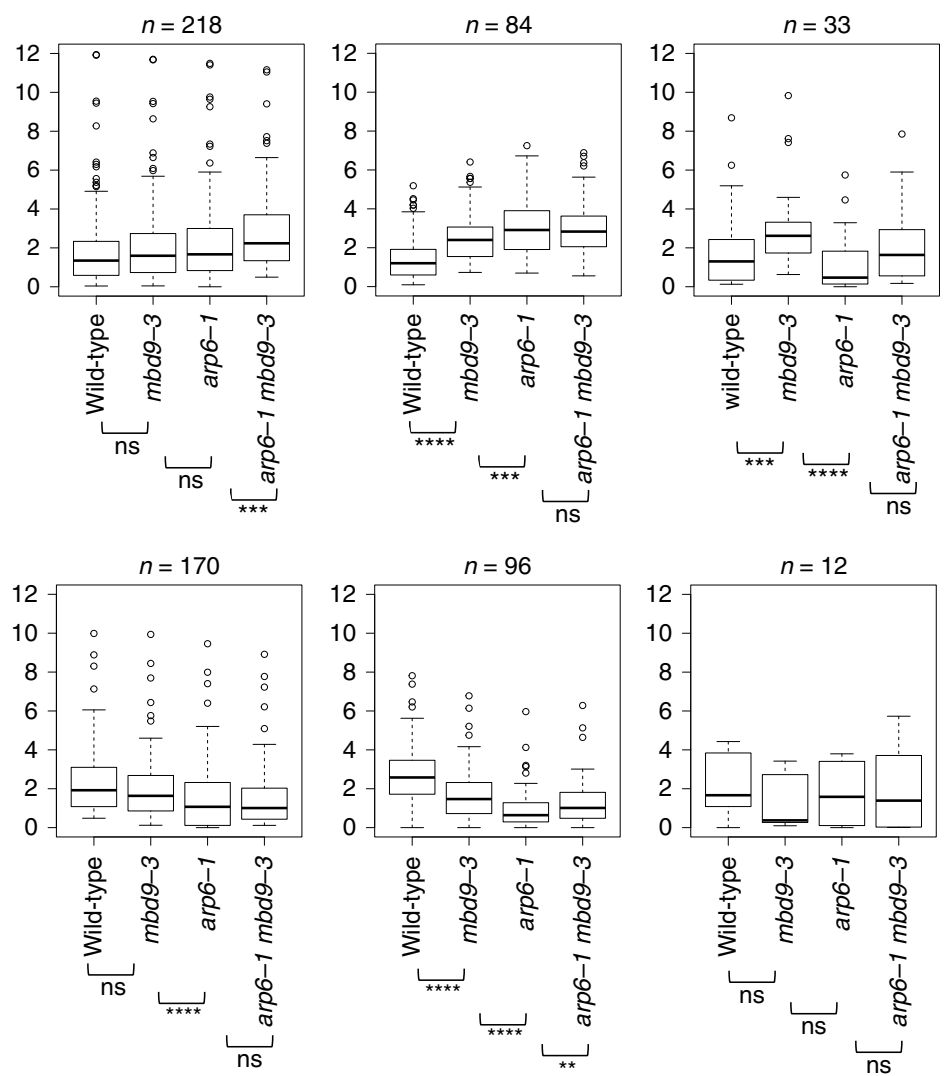
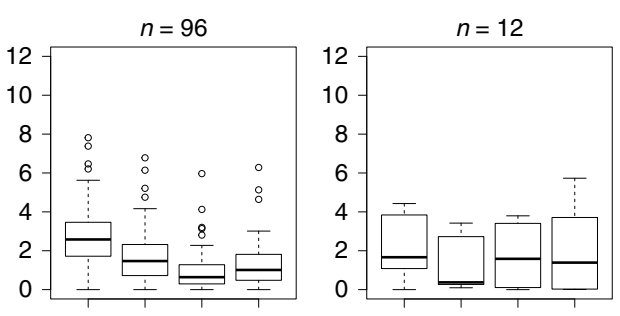

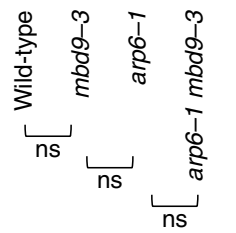

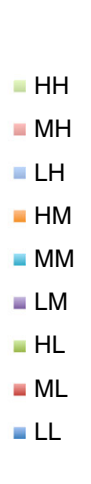

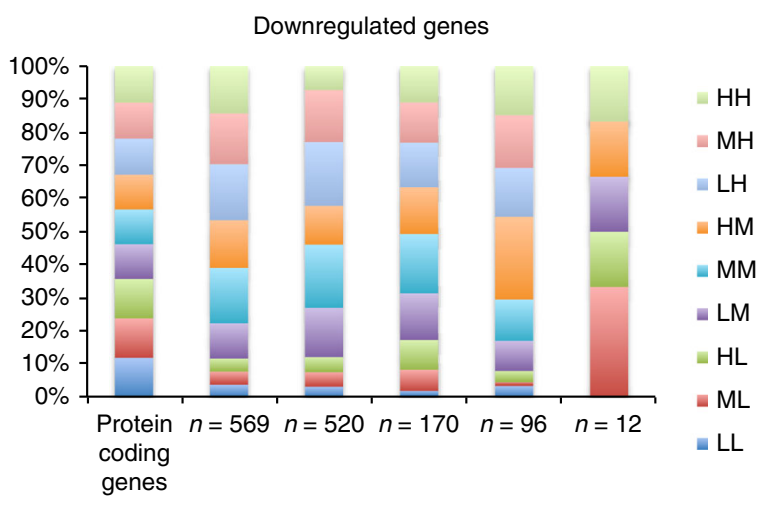

protein-coding genes revealed two major MBD9 peaks, one over nucleosome-depleted regions just upstream of the TSS and a smaller peak just $3^{\prime}$ of the transcription end site (TES) (Fig. 4d). At the $5^{\prime}$ end, the MBD9 peaks overlap with the ATAC-seq peaks and are just upstream of the H2A.Z peak (Fig. 4d, e).

We further investigated MBD9 occupancy in relation to H2A.Z distribution. When plotting H2A.Z over MBD9-occupied genes, we detected a loss of H2A.Z at the $5^{\prime}$-ends of genes in the mbd9-3 mutant (Fig. 4f). To investigate whether the loss of H2A.Z occurs over a specific subset of genes, we ordered MBD9-occupied genes based on H2A.Z occupancy in the wild type using $k$-means 
Fig. 3 RNA-seq and H2A.Z levels in wild type and mutants. a Overlap between significantly $\left(\log _{2} \geq 1, F D R \leq 0.05\right)$ up-regulated and $\mathbf{b}$ down-regulated genes for each mutant vs wild-type control. Boxplots of average normalized RNA-seq reads $\log _{2}(F P K M+1)$ represent different classes of overlapping and non-overlapping $\mathbf{c}$ up-regulated and $\mathbf{d}$ down-regulated genes based on intersection. Center line indicates the median, upper and lower bounds represent the 75th and the 25th percentile, respectively, whiskers indicate the minimum and the maximum, and outliers are represented as circles. Unpaired twosamples Wilcoxon test was used to determine significance: ns $p$ value $>0.05,{ }^{\star} p$ value $\leq 0.05,{ }^{\star \star} p$ value $\leq 0.01,{ }^{\star \star \star} p$ value $\leq 0.001,{ }^{\star \star \star \star} p$ value $\leq 0.0001$. e Intersection of up-regulated and down-regulated genes with nine classes of genes characterized by their distribution of H2A.Z in wild type at TSS and gene body. Labels H-high, M-medium, L-low refer to levels of H2A.Z at TSS (first letter) and gene body (second letter). Source data are provided as Source Data file

clustering $(n=5)$ to reflect typical patterns of H2A.Z occupancy in Arabidopsis $^{17}$ (Fig. 4g). This analysis revealed that MBD9 occupies genes with various H2A.Z localization patterns, including genes with low H2A.Z throughout (cluster 2, Fig. 4g) and genes containing H2A.Z over gene bodies (clusters 1 and 5, Fig. $4 \mathrm{~g}$ ). However, the locations that were most sensitive to the loss of H2A.Z in the mbd9-3 mutants were those that contain H2A.Z predominately over their $5^{\prime}$-ends (clusters 3 and 4, Fig. $4 \mathrm{~g}$, ratio plots). These regions (clusters 3 and 4) contain low levels of $\mathrm{H} 3 \mathrm{~K} 27 \mathrm{me}$. This is in contrast to the arp6-1 and the arp6-1 mbd9-3 mutants, where H2A.Z loss occurred throughout all MBD9-occupied genes, with the greatest loss observed in the double mutant (Fig. 4g).

MBD9-enriched genes contain specialized chromatin features. To determine if specific chromatin features mark the regions that are enriched with MBD9, we analyzed ATAC-seq data from wildtype plants and observed that MBD9-occupied genes tended to have more open chromatin than either an equivalent set of genes with similar expression levels, or all protein-coding genes (Fig. 5a, b). We also investigated preferences for histone modifications using previously published and generated data ${ }^{62-64}$ from equivalent tissues and stages of development (Supplementary Note). MBD9-occupied genes contained higher levels of H3K4me3 when compared to all protein-coding genes and the H3K4me3 signal was broader when compared to a control set of genes (Fig. 5c). A similar relative enrichment was also true for histone acetylation levels (Fig. 5c), whereas MBD9-occupied genes were depleted of $\mathrm{H} 3 \mathrm{~K} 27 \mathrm{me} 3$ when compared to gene averages (Fig. $5 \mathrm{c}$ ). This general trend was also true for regions depleted of H2A.Z in $m b d 9-3$, as depicted in the heatmap ordered by expression level at all protein-coding genes (Supplementary Fig. 8). Regions that lost H2A.Z most in $m b d 9-3$ tended to occur predominantly at the $5^{\prime}$-ends of genes. These regions also contained high levels of activating histone modifications (H3K4me3, $\mathrm{H} 3 \mathrm{Ac}$ ) and low levels of H3K27me3, and coincided with MBD9occupied peaks and open chromatin. In agreement, a related study also identified an enrichment of histone acetylation marks at MBD9-dependent H2A.Z sites ${ }^{54}$.

Altogether, these results suggest that MBD9 preferentially localizes to the open chromatin regions of actively transcribing genes, which contain high levels of $\mathrm{H} 3 \mathrm{~K} 4 \mathrm{me} 3$ and histone acetylation and are different from genes controlled by the Polycomb Group Complex.

\section{Discussion}

H2A.Z has been implicated in a wide variety of functions, including transcription, meiosis, and DNA damage ${ }^{52}$. How it is specifically targeted to the locations where these events occur is poorly understood. In this study we have characterized the interacting partners of Arabidopsis ARP6, a homolog of Arp6 found in the yeast SWR1-C involved in H2A.Z deposition. We identified nine Arabidopsis proteins that are homologous to subunits common to the yeast (SWR1) and mammalian (SRCAP) complexes, ARP6, PIE1, RIN1, RIN2, ARP4, SWC4, SWC2, YAF9A, and SEF. We further identified several additional ARP6interacting partners, TRA1A, TRA1B, MBD9, and four members of the Alfin1-like family (AL4, AL5, AL6, and AL7). These Alfin1like-containing proteins recognize $\mathrm{H} 3 \mathrm{~K} 4 \mathrm{me} 3$ histones ${ }^{57}$, suggesting that they play a role in recruiting the SWR1 complex to $\mathrm{H} 3 \mathrm{~K} 4 \mathrm{me} 3$ regions. The ARP6-interacting partners identified in our study are in agreement with a related study involving ARP6TAP purification followed by mass spectrometry ${ }^{54}$. However, there were a few differences. Specifically, we did not enrich for H2A.Z or ACT1 in our mass spec data, perhaps reflecting different methods of isolation, while we did identify TRA1A and AL4 as ARP6-interacting partners that were not reported by Sijacic et al. ${ }^{54}$.

We focused on characterizing the interaction between ARP6 and MBD9. We identified interacting partners for MBD9 and showed that it interacted with most components of the SWR1 complex, including PIE1, RIN1, ARP4, RIN2, SWC2, ARP6, SWC4, YAF9A, and SEF. MBD9 also interacted with TRA1A and TRA1B, further implicating histone acetylation in H2A.Z deposition. Additionally, we detected high enrichment of the ISWI chromatin remodeling complex in our MBD9 IP, which was not seen in the ARP6 IP-mass spec. The ISWI remodeling complex interacts with other chromatin remodelers ${ }^{65}$, MADS proteins $^{66}$, and DTT-containing proteins ${ }^{67}$. MBD9 was among the reported interacting partners of CHR17, independently validating our results ${ }^{65}$. However, only two other SWR1 complex components (ARP4 and RIN1) were detected in the same CHR17 IP-mass spec study. Since we did not detected preferential enrichment between CHR11/17 and ARP6 in our data, this suggests that MBD9 interacts with the two complexes separately. Our analysis of the genetic interactions between $m b d 9-3$ and arp6-1 suggests that, despite the more severe developmental phenotype of the double mutant, ARP6 is epistatic to MBD9 regarding flowering time. These two observations are in agreement since flowering time is controlled by a particular set of genes, whereas H2A.Z deposition is globally affected in our mutants. The stronger developmental phenotype of the double mutant could have two plausible explanations: (1) multiple pathways are involved or (2) since the double mutant closely resembles pie1-2 (a mutation affecting the catalytic subunit of the SWR1 complex), the phenotype results from MBD9 and ARP6 being part of the SWR1 complex, where the complex may be partly functional without one of the components but not without both. Future work will discriminate between these possibilities. Our genomic observations, H2A.Z ChIP-seq data, and transcriptome analysis revealed that MBD9 and ARP6 share many common targets, but the mbd9-3 mutant shows a weaker phenotype than arp6-1. We observed that MBD9 is required for the deposition of H2A.Z at a subset of arp6-1 dependent loci, similar to what was reported by Sijacic et al. ${ }^{54}$. We also identified a subset of genes that require both ARP6 and MBD9 for both their expression and for H2A.Z deposition, suggesting the two proteins act redundantly in some cases. Additionally, we determined the genome-wide occupancy of MBD9 and showed that it 
a

MBD9-flag/wild-type flag

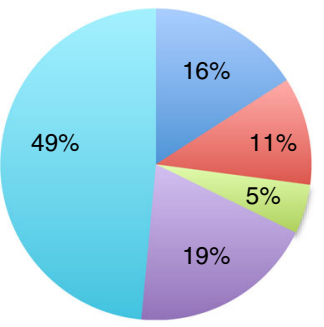

TSS Exon Intron
Control

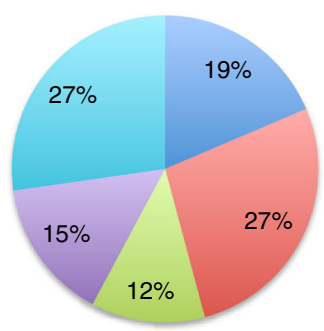

b

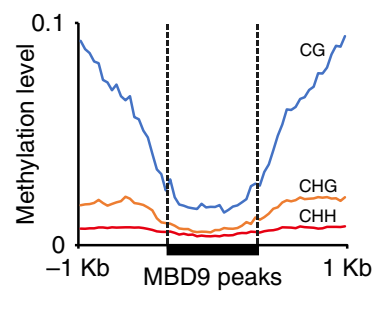

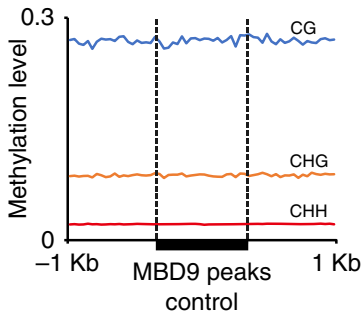

control

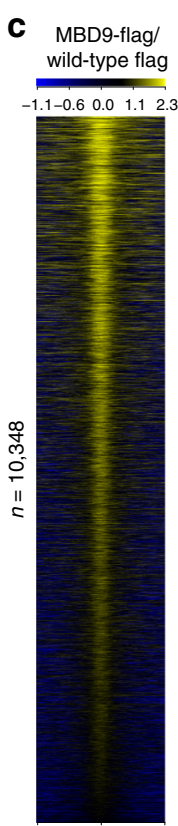

-1000 Center $1000-1000$ Center $1000-1000$ Center 1000

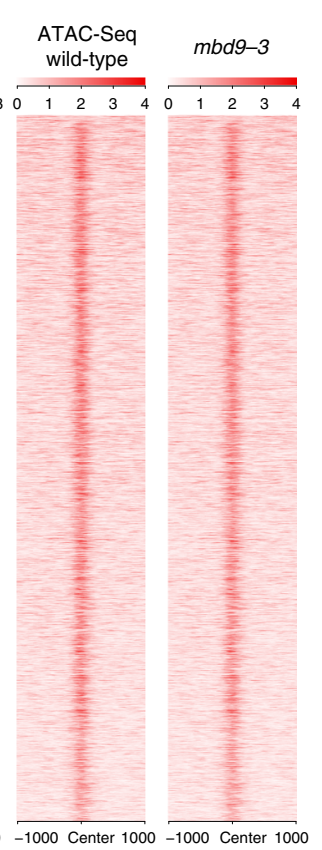

d

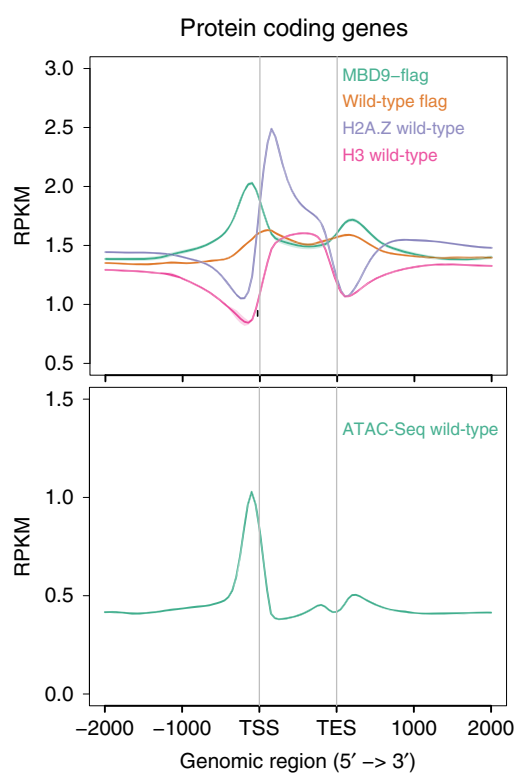

e

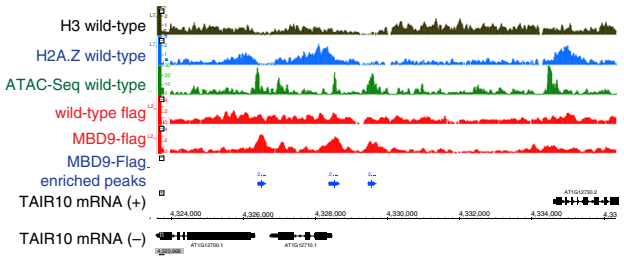

$\mathbf{f}$

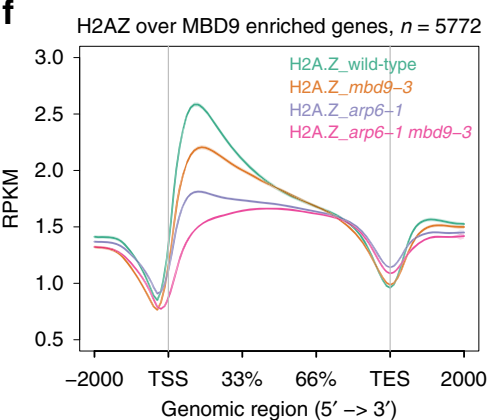

g

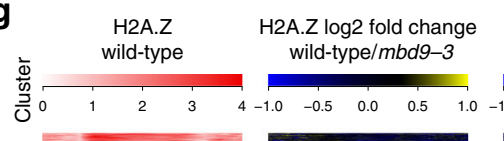

Wild-type/arp6-1
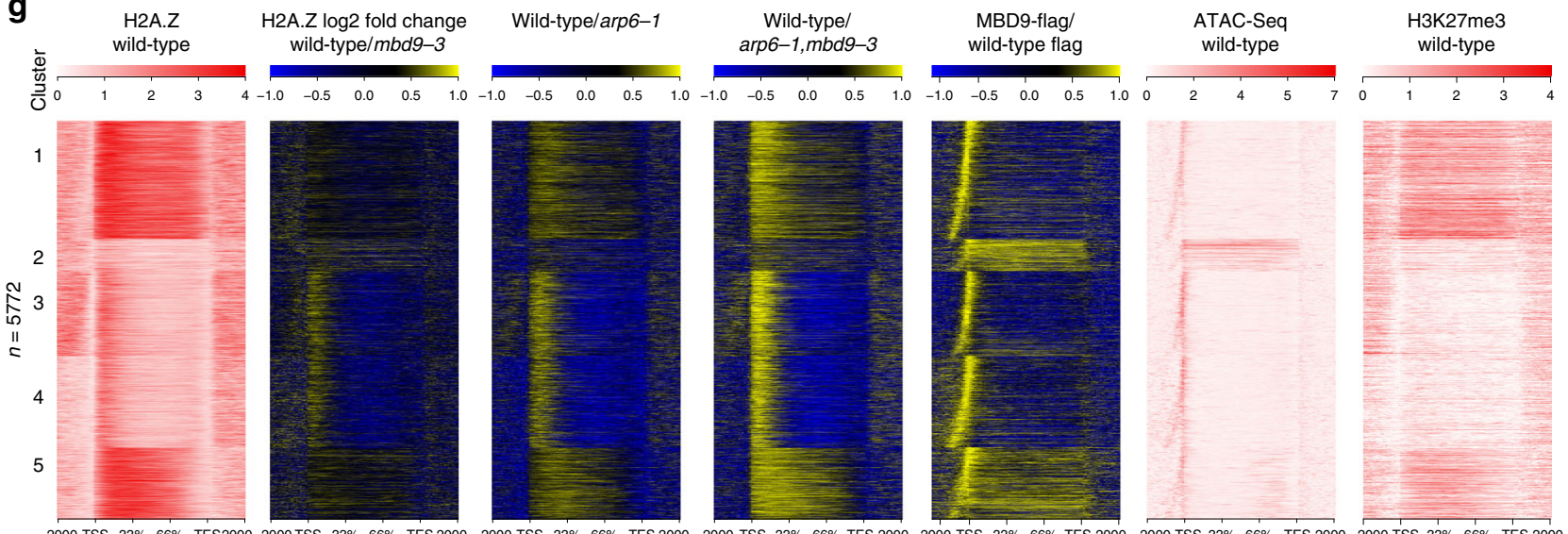

Fig. 4 Characterization of MBD9 chromatin binding. a Genomic distribution of MBD9-enriched peaks vs wild type (mac2 peak caller, $q$ value $<0.01$ ). b Average distribution of DNA methylation levels in $\mathrm{CG}, \mathrm{CHG}$, and $\mathrm{CHH}$ contexts in wild type over MBD9-occupied peaks and MBD9 control peaks. c Heatmap of normalized MBD9 signal ( $\log _{2}$ RPKM) over wild-type control and normalized ATAC-seq signal (RPKM) from merged replicates in wild type and mbd9-3 mutant over MBD9-occupied peaks. d Upper: Distribution of normalized signal (RPKM) for MBD9-3xFlag ChIP-seq, wild-type Flag ChIP-seq control, H2A.Z, and H3 in wild type over protein-coding genes. Lower: Distribution of normalized signal (RPKM) for ATAC-seq in wild type over proteincoding genes. e Snapshot representing normalized read counts (RPKM) for MBD9-3xFlag ChIP-seq, wild-type Flag ChIP-seq control, ATAC-seq, H2A.Z, and H3. Signal for ATAC-seq, H2A.Z, and H3 is a merge of two biological replicates. $\mathbf{f}$ Distribution of normalized H2A.Z ChIP-seq signal (RPKM) from merged replicates in wild type, mbd9-3, arp6-1, and arp6-1 mbd9-3 over MBD9-occupied genes. $\mathbf{g}$ Heatmap ordered based on $k$-means clustering of H2A.Z ChIP-seq signal in wild type (cluster $n=5$ ) at MBD9-occupied genes. Shown are corresponding H2A.Z ratio plots for wild type over mbd9-3, arp6-1, and arp6-1 mbd9-3 mutants and MBD9-3X Flag ChIP-seq over wild-type flag control in log 2 RPKM, ATAC-seq in wild type in RPKM, and H3K27me3 levels in wild type in RPKM. Source data are provided as Source Data file 
a

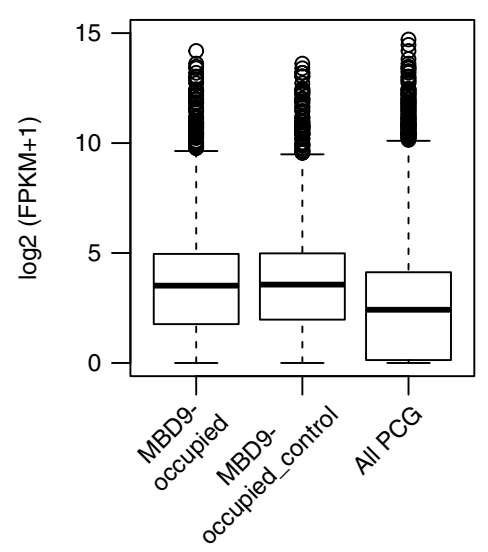

C
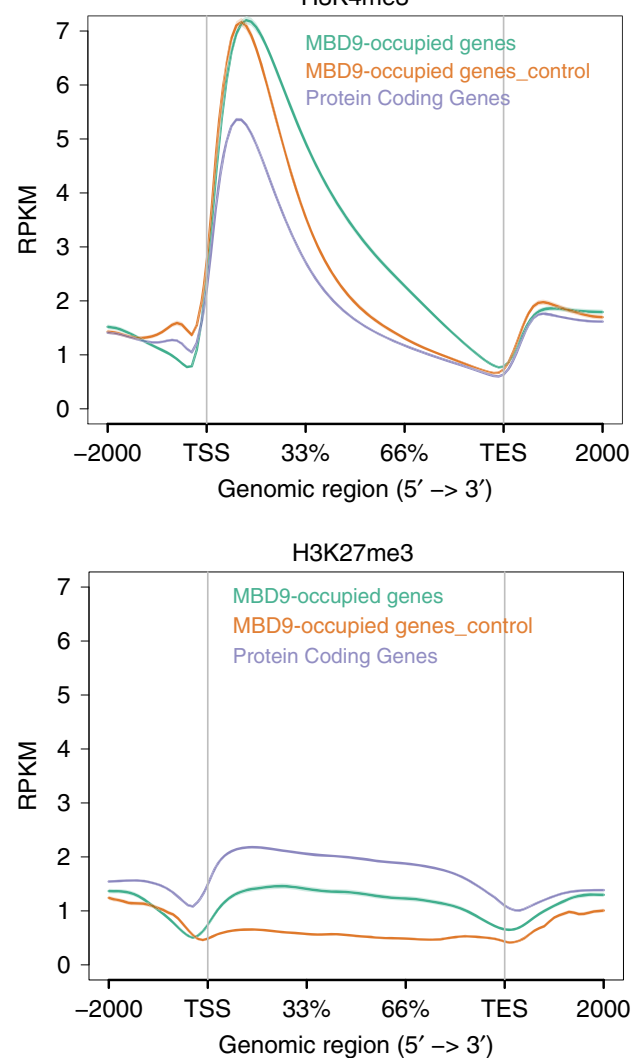

b

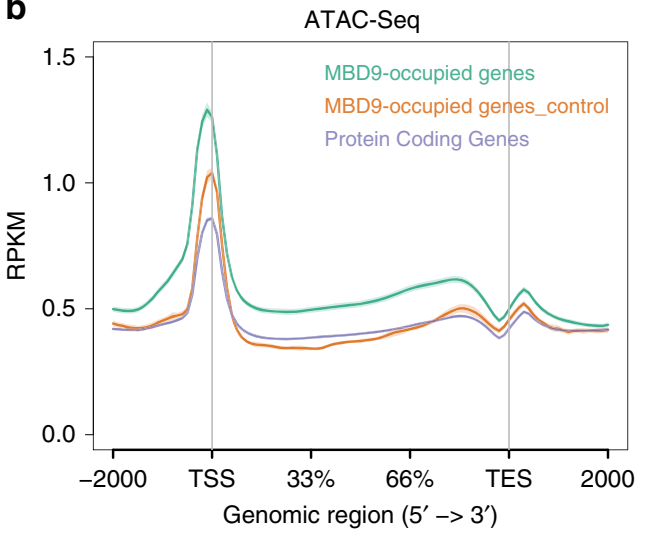

$\mathrm{H} 3 \mathrm{Ac}$

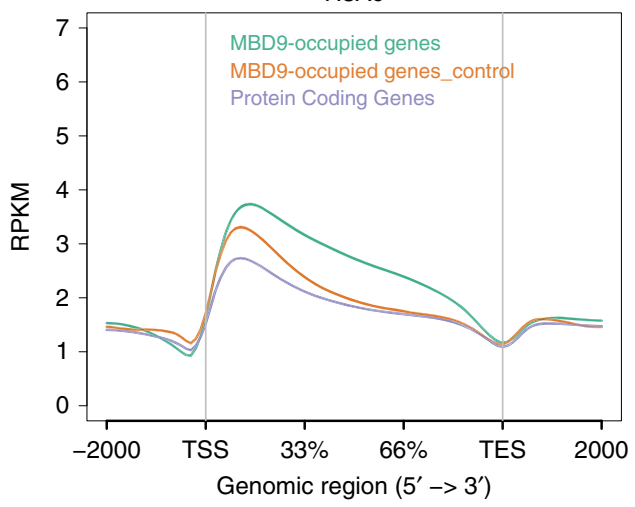

H3

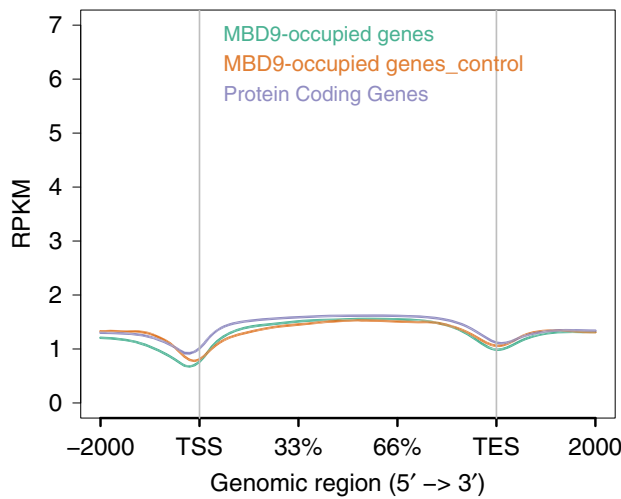

Fig. 5 Chromatin features at MBD9-occupied genes. a Boxplots of average expression levels $\log _{2}$ (FPKM + 1) from RNA-seq in wild type for MBD9occupied genes, MBD9 control genes, and protein-coding genes. Center line indicates the median, upper and lower bounds represent the 75th and the 25th percentile, respectively, whiskers indicate the minimum and the maximum, and outliers are represented as circles. b Distribution of normalized ATAC-seg levels (RPKM) over MBD9-occupied genes, MBD9 control genes, and protein-coding genes. c Distribution of normalized levels (RPKM) for H3K4me3, H3Ac, H3K27me3, and H3 ChIP-seq in MBD9-occupied genes, MBD9 control genes, and protein-coding genes

preferentially localized to the open chromatin regions of promoters and the transcription termination sites of active genes that have high levels of activating histone modifications (H3K4me3 and histone acetylation) and did not localize to genes repressed by the Polycomb Group Complex. These data suggest that the MBD9-containing SWR1 complex promotes H2A.Z deposition at the $5^{\prime}$ ends of highly expressed genes.

Regarding the interaction of MBD9 with the SWR1 complex, three possibilities exist based on our genetic and genomic observations: (1) MBD9 is always a member of the SWR1 complex but not always required, (2) MBD9 is part of a subset of all SWR1 complexes in Arabidopsis, and (3) MBD9 only transiently interacts with the SWR1 complex. Based on size exclusion chromatography of ARP6-containing SWR1 complex in the mbd9 mutant, Sijacic et al. ${ }^{54}$ concluded that MBD9 is not a core subunit of the Arabidopsis SWR1 complex. However, as noted by the authors, this does not exclude the possibility that MBD9 is part of a small subset of all SWR1 complexes in Arabidopsis ${ }^{54}$. Given that we detected most components of the SWR1 complex in our MBD9 IP-mass spec, our genetic and genomic data best fit the model that MBD9 is part of a subset of the SWR1-containing complexes.

Surprisingly, although we detected many genes that lost $\mathrm{H} 2 \mathrm{~A} . \mathrm{Z}$ in the mbd9-3 mutant, this loss did not result in major transcriptional changes. This may be because the loss of H2A.Z in the 
mbd9-3 mutant occurs over genes with low H2A.Z over gene bodies and several studies suggest that genes changing most upon loss of H2A.Z are the ones bearing H2A.Z over the gene body ${ }^{17,23,24}$. One plausible explanation for the lack of transcriptional response at these regions in the $m b d 9-3$ mutant is redundancy. It is possible that redundant mechanisms are present at housekeeping genes to maintain their high expression and losing H2A.Z is not enough to perturb their activity. In support, H2A.Z is proposed to have redundant roles with chromatin remodeling complexes in yeast ${ }^{68}$. Interestingly, we identify components of the ISWI chromatin remodeling family as interacting partners of MBD9 in our IP. Additionally, CHR11/17 mutants have defects in flowering time and decreased levels of FLC expression ${ }^{69}$. Perhaps in addition to promoting H2A.Z deposition, MBD9 promotes low nucleosome occupancy at genes containing H2A.Z at +1 by interacting with chromatin remodelers to maintain these loci in a transcriptionally permissive state. In support, a recent study of FORGETTER1, a protein involved in heat-shock memory response, was found to interact with chromatin remodelers including CHR11/17 to maintain low nucleosome occupancy of genes in non-inductive conditions ${ }^{65}$. In Arabidopsis, the ISWI remodeling complex is required for the regular spacing of nucleosomes downstream of the +1 border nucleosome $\mathrm{e}^{70}$. Interestingly, many highly expressed genes in the CHR11/17 mutant have defects in nucleosome positioning, yet their transcription is unaffected ${ }^{70}$. This finding supports our hypothesis that redundant mechanisms maintain high expression of housekeeping genes. Higher order mutants between the SWR1 complex and chromatin remodelers are required to test this hypothesis.

In yeast, the SWR1 complex preferentially binds to nucleosomedepleted regions ${ }^{42,43}$. Consistent with the role of MBD9 in the SWR1 complex, we found that MBD9 also localizes to nucleosomedepleted regions, in close proximity to $\mathrm{H} 2 \mathrm{~A} . \mathrm{Z}$ deposited at the $5^{\prime}$ ends of gene bodies. These regions are devoid of DNA methylation, consistent with the prediction that the methyl-binding domain of MBD9 does not recognize methylated sequences ${ }^{61}$.

The genes bearing MBD9 also contain high levels of histone acetylation, consistent with the observation that histone acetylation in yeast promotes H2A.Z deposition. We also observed enrichment of three of the four common NuA4 histone acetylation complex subunits, SWC4, YAF9, and ARP4, in our IP-mass spec. We also identified two homologs of the yeast Tral proteins, TRA1A and TRA1B, in both ARP6 and MBD9 IPs. Tral is a component of the yeast NuA4 complex ${ }^{50}$. In Arabidopsis, TRA1B interacts with SWC4, a component of the predicted NuA4 complex $^{71}$, and both TRA1A and TRA1B are components of the Arabidopsis SAGA acetyltransferase complex ${ }^{72}$. This suggests that various acetyltransferase complexes may be promoting H2A.Z deposition via histone acetylation. Since we did not detected the NuA4 scaffold or the catalytic subunit in our IP, it is unlikely that the ARP6-containing SWR1 complex represents a fusion of the Arabidopsis SWR1 and NuA4 complexes, similar to the mammalian TIP60 complex; rather, the TRA1A and TRA1B subunits may function to bridge the acetyltransferases with the SWR1 complex.

In summary, our study identified components of the SWR1 complex in Arabidopsis and shed light on the role of MBD9 in promoting H2A.Z deposition at a subset of highly expressed genes. Further work addressing the function of the H2A.Z histone variant should reveal the nature of its dependency on factors such as histone acetyltransferase and chromatin remodeling complexes for carrying out its varied functions across the genome.

\footnotetext{
Methods

Plant materials. All Arabidopsis plants used in this study were of the Col-0 ecotype
} and were grown at $22^{\circ} \mathrm{C}$ under long-day conditions ( $16 \mathrm{~h}$ light, $8 \mathrm{~h}$ dark). For the short-day flowering time experiment, plants were grown at $22^{\circ} \mathrm{C}(16 \mathrm{~h}$ dark, $8 \mathrm{~h}$ light). The following Arabidopsis mutant lines were used: mbd9-3 (SALK_039302), arp6-1 (Sail_599_G03), and pie1-2 (SALK_003776C).

Quantitative real time PCR. Total RNA was prepared from whole seedlings (14 DAG) using the RNA Prep Pure Plant Kit (Tiangen Biotech, Beijing, China). Two micrograms of RNA was used for the preparation of cDNA using PrimeScript Reagent Kit with gDNA eraser (Takara, Japan). Signal detection, quantification, and normalization were done using Quant Studio 6 proprietary Software (Life Technologies, USA).

Co-immunoprecipitation. An anti-MBD9 polyclonal antibody was raised in rabbits using the 1941-2176 aa MBD9 peptide as an antigen. The antibody was coupled to magnetic beads using the Dynabeads antibody coupling kit (Invitrogen, USA) following the manufacturer's recommendations; $7 \mu \mathrm{L}$ of $3.12 \mathrm{mg} \mathrm{mL}^{-1}$ were used per sample. Approximately $2 \mathrm{~g}$ of floral tissue was ground in liquid nitrogen, $10 \mathrm{~mL}$ IP buffer (50 mM Tris pH 7.6, $150 \mathrm{mM} \mathrm{NaCl}, 5 \mathrm{mM} \mathrm{MgCl}_{2}, 10 \%$ glycerol, $0.1 \% \mathrm{NP}-40,0.5 \mathrm{mM}$ DTT, $1 \mu \mathrm{g} \mu \mathrm{L}^{-1}$ pepstatin, $1 \mathrm{mM}$ PMSF, 1 protease inhibitor cocktail tablet (Roche, 14696200)) was added, the mix was incubated on ice for 20 min, and spun at $4000 \mathrm{~g}$ for $10 \mathrm{~min}$ at $4{ }^{\circ} \mathrm{C}$. The supernatant was filtered through a double layer of Miracloth. Anti-MBD9 antibody coupled to magnetic beads was added and incubated for $2 \mathrm{~h}$ at $4{ }^{\circ} \mathrm{C}$ with gentle rotation. Beads were washed six times with IP buffer, $5 \mathrm{~min}$ each wash at $4^{\circ} \mathrm{C}$ with rotation, and then boiled. Western blotting and detection were performed with anti-Myc 9E10 mouse monoclonal antibody (Thermo Fisher Scientific, MA1-980) with 1:1000 dilution.

RNA-seq and analysis. Total RNA was extracted from 13 DAG shoots grown on $1 \%$ MS supplemented with $1 \%$ sucrose under long-day conditions. Four replicates, grown on separate plates, were collected for each genotype. RNA was extracted using the Direct-zol RNA Miniprep kit (Zymo). For RNA-seq, $1 \mu \mathrm{g}$ of total RNA was used to prepare libraries using the TruSeq Stranded mRNA-Seq kit (Illumina) Reads were aligned to TAIR10 using Tophat by allowing up to two mismatches and mapping only to one location. FPKM values and differential gene expression were analyzed using Cufflinks with default settings. GO term enrichment was determined using AgriGO.

Epitope-tagged transgenic ARP6 and MBD9 lines. Full-length genomic DNA fragments containing $\sim 1 \mathrm{~kb}$ (MBD9) or $\sim 1.5 \mathrm{~kb}$ (ARP6) of promoter sequence, together with genomic gene sequences up to the annotated/major stop codon of MBD9 and ARP6 were amplified using PCR (primers are listed in Supplementary Table 1). The PCR product was cloned into the $\mathrm{pENTR/D}$ vector (Invitrogen) and delivered into a modified pEG containing 3X FLAG and 9X MYC tags using the LR reaction kit (Invitrogen). The $\mathrm{pEG}$ destination vectors were transformed into Agrobacterium strain AGL0, followed by transformation using the floral dip method.

Affinity purification and mass spectrometry. Approximately $10 \mathrm{~g}$ of flowers from transgenic lines expressing T3 ARP6-3xFLAG or T4 MBD9-3xFLAG and from Col-0 plants as negative controls were ground to a fine powder using a RETCH homogenizer (3 min @ $30 \mathrm{~Hz} \mathrm{~min}^{-1}$ ) and suspended in $30 \mathrm{~mL}$ of IP buffer Tissue was further dounce homogenized until lump-free, then centrifuged for 20 $\min$ at $4000 \mathrm{~g}$ and $4{ }^{\circ} \mathrm{C}$. The lysate was filtered through two layers of Miracloth. Supernatant was incubated with $200 \mu \mathrm{L}$ anti-FLAG M2 magnetic beads (M8823, Sigma), at $4^{\circ} \mathrm{C}$ for $2 \mathrm{~h}$. The bead-bound complex was washed once with $10 \mathrm{~mL} \mathrm{IP}$ buffer, then four times ( $5 \mathrm{~min}$ rotating at $4{ }^{\circ} \mathrm{C}$ with $1.5 \mathrm{~mL}$ IP buffer), followed by a final wash with IP buffer without NP-40. The FLAG-IP was eluted twice with 300 $\mu \mathrm{L} 250 \mu \mathrm{g} \mathrm{mL}^{-1} 3 \mathrm{X}$ FLAG peptides (Sigma, F4799) in TBS (50 mM Tris-Cl, pH 7.5, $150 \mathrm{mM} \mathrm{NaCl}$ ), mixing for $15 \mathrm{~min}$ at $4^{\circ} \mathrm{C}$. The eluted protein complexes were precipitated by trichloroacetic acid and subjected to mass spectrometric analyses. In the case of ARP6-9xMyc and MBD9-9xMyc, monoclonal 9E10 coupled to magnetic beads was used (88842, Pierce). The bead-bound complexes were washed six times with $1 \mathrm{~mL}$ of IP buffer. For each wash, the beads were rotated at $4{ }^{\circ} \mathrm{C}$ for 5 min. For ARP6-9xMyc, proteins were released from the Streptavidin beads by 3C cleavage overnight at $5{ }^{\circ} \mathrm{C}$. 3C-GST was removed by incubation with GST couple magnetic beads (Pierce, USA). For MBD9-9xMyc, proteins were eluted twice with $100 \mu \mathrm{L} 8 \mathrm{M}$ Urea in $50 \mathrm{mM}$ Tris $\mathrm{pH} 8.5$, mixing for $15 \mathrm{~min}$ at $37^{\circ} \mathrm{C}$. The supernatant was TCA precipitated.

Genome-wide ChIP sequencing and library generation. For histone modification ChIPs, about $0.5 \mathrm{~g}$ of shoots were collected from 13 DAG seedlings grown on $1 \%$ MS plates supplemented with $1 \%$ sucrose under long-day conditions. Samples were crosslinked in vitro, sheared using Bioruptor Plus (Diagenode). Libraries were generated with NuGEN Ovation Ultra Low System V2 kit, according to the manufacturer's instructions, and were sequenced on an Illumina HiSeq 4000 instrument. H2A.Z ChIP assays were performed using polyclonal antibodies specific to Arabidopsis H2A.Zs HTA11 and HTA9. The antibodies were produced in rabbits and further purified using the peptides described in Deal et al. ${ }^{57}$. For each ChIP we used $4 \mu \mathrm{L}$ of $3.34 \mathrm{mg} \mathrm{mL}^{-1}$. Abcam1791 (Abcam) antibody was used for 
H3 ChIP assays ( $10 \mu \mathrm{L}$ of $0.5 \mathrm{mg} \mathrm{mL}^{-1}$ ) and active motif 39140 (Active Motif) was used for pan-acetyl $\mathrm{H} 3 \mathrm{ChIP}$ assays $\left(5 \mu \mathrm{L}\right.$ of $\left.1 \mathrm{mg} \mathrm{mL}^{-1}\right)$.

For MBD9, about $5 \mathrm{~g}$ of 13 DAG T4 MBD9-3XFLAG and Col-0 seedlings grown on $1 \%$ MS were fixed in vivo using vacuum infiltration as follows. Protein-protein crosslinking was performed first using $1.5 \mathrm{mM}$ EGS in $1 \times$ phosphate-buffered saline (PBS) for $10 \mathrm{~min}$ followed by three washes with PBS. Next, tissue was fixed with $1 \%$ formaldehyde in $1 \times$ PBS for 20 min. Crosslinking was stopped with $0.125 \mathrm{M}$ glycine in $1 \times$ PBS and the seedlings were vacuum infiltrated for $5 \mathrm{~min}$. Tissue was patted dry and frozen at $-80^{\circ} \mathrm{C}$. Tissue was homogenized to a fine powder using a RETCH homogenizer (2 min @ $30 \mathrm{~Hz}$ min ${ }^{-1}$ ) and resuspended with Nuclear Isolation Buffer (50 mM Hepes, $1 \mathrm{M}$ Sucrose, 5 $\mathrm{mM} \mathrm{KCl}, 5 \mathrm{mM} \mathrm{MgCl} 2,0.6 \%$ Triton X-100, $0.4 \mathrm{mM}$ PMSF, $5 \mathrm{mM}$ Benzamidine, $1 \times$ protease inhibitor cocktail tablet (Roche, 14696200). Lysate was filtered through one layer of Miracloth and centrifuged for $20 \mathrm{~min}$ at $2880 \mathrm{~g}$ and $4^{\circ} \mathrm{C}$. The pellet was resuspended with $1 \mathrm{~mL}$ Extraction buffer $2(0.25 \mathrm{M}$ sucrose, $10 \mathrm{mM}$ Tris- $\mathrm{HCl} \mathrm{pH} 8$, $10 \mathrm{mM} \mathrm{MgCl} 2,1 \%$ Triton X-100, 5 mM BME, 0.1 mM PMSF, 5 mM Benzamidine, and $1 \times$ protease inhibitor cocktail tablet (Roche, 14696200)), followed by centrifugation for $10 \mathrm{~min}$ at $12,000 \mathrm{~g}$ and $4{ }^{\circ} \mathrm{C}$. The pellet was then resuspended in $500 \mu \mathrm{L}$ Extraction buffer $3\left(1.7 \mathrm{M}\right.$ sucrose, $10 \mathrm{mM}$ Tris- $\mathrm{HCl} \mathrm{pH} 8,2 \mathrm{mM} \mathrm{MgCl}_{2}$, $0.15 \%$ Triton X-100, $5 \mathrm{mM}$ BME, $0.1 \mathrm{mM}$ PMSF, $5 \mathrm{mM}$ Benzamidine, $1 \times$ protease inhibitor cocktail tablet (Roche, 14696200), layered over $500 \mu \mathrm{L}$ extraction buffer 3 and centrifuged for $1 \mathrm{~h}$ at $12,000 \mathrm{~g}$ and $4{ }^{\circ} \mathrm{C}$. The pellet was lysed with $400 \mu \mathrm{L}$ Nuclei Lysis Buffer on ice (50 mM Tris pH 8, $10 \mathrm{mM}$ EDTA, 1\% SDS, $0.1 \mathrm{mM}$ PMSF, 5 $\mathrm{mM}$ Benzamidine, $1 \times$ protease inhibitor cocktail tablet (Roche, 14696200). To lysed nuclei, $1.7 \mathrm{~mL}$ of ChIP Dilution Buffer (1.1\% Triton X-100, $1.2 \mathrm{mM}$ EDTA, $16.7 \mathrm{mM}$ Tris $\mathrm{pH} 8,167 \mathrm{mM} \mathrm{NaCl}, 0.1 \mathrm{mM}$ PMSF, $5 \mathrm{mM}$ Benzamidine, $1 \times$ protease inhibitor cocktail tablet (Roche, 14696200) was added and DNA was sheared on a Bioruptor Plus (Diagenode) (30 s ON/30 s OFF, Max power, $17 \mathrm{~min}$ at $4{ }^{\circ} \mathrm{C}$ ). Sheared chromatin was centrifuged twice at max speed for $10 \mathrm{~min}$ at $4^{\circ} \mathrm{C}$. The supernatant was further diluted with ChIP dilution buffer up to $4 \mathrm{~mL}$. One hundred microliters of sample were saved as input and the rest was split into $2 \mathrm{~mL}$ DNA LoBind tubes (Eppendorf, D1579630) and $10 \mu \mathrm{L}$ of $1 \mathrm{mg} \mathrm{mL}^{-1}$ anti-Flag antibody was added (F1804; Sigma-Aldrich). After incubation overnight with rotation at $4{ }^{\circ} \mathrm{C}, 50 \mu \mathrm{L}$ Dynabeads (equal mix of Protein A and G; Invitrogen $10004 \mathrm{D} / 10002 \mathrm{D}$ ) was added to chromatin and incubated for an additional $2 \mathrm{~h}$. The magnetic beads were washed with $1 \mathrm{~mL}$ of the following buffers for $5 \mathrm{~min}$ rotating at $4{ }^{\circ} \mathrm{C}$ : $2 \times$ with low salt buffer $(150 \mathrm{mM} \mathrm{NaCl}, 0.2 \%$ SDS, $0.5 \%$ Triton X-100, 2 mM EDTA, $20 \mathrm{mM}$ Tris $\mathrm{pH}$ 8), $1 \times$ high salt buffer $(200 \mathrm{mM} \mathrm{NaCl}, 0.2 \%$ SDS, $0.5 \%$ Triton X-100, $2 \mathrm{mM}$ EDTA, $20 \mathrm{mM}$ Tris pH 8), $1 \times \mathrm{LiCl}$ Wash Buffer $(250 \mathrm{mM}$ $\mathrm{LiCl}, 1 \%$ Igepal, $1 \%$ sodium deoxycholate, $1 \mathrm{mM}$ EDTA, $10 \mathrm{mM}$ Tris $\mathrm{pH} 8$ ), and $1 \times$ with TE buffer (10 mM Tris $\mathrm{pH} 8,1 \mathrm{mM}$ EDTA). The immunocomplex was eluted from the beads twice with $250 \mu \mathrm{L}$ elution buffer $(1 \%$ SDS, $10 \mathrm{mM}$ EDTA, $0.1 \mathrm{M}$ $\mathrm{NaHCO}_{3}$ ), incubating for $20 \mathrm{~min}$ with shaking at $65^{\circ} \mathrm{C}$. Four hundred microliters of elution buffer was added to the input samples. A total of $20 \mu \mathrm{L} 5 \mathrm{M} \mathrm{NaCl}$ was added to each tube; the crosslink was reversed by incubation at $65^{\circ} \mathrm{C}$ overnight. Residual protein was degraded with $20 \mu \mathrm{g}$ Prot $\mathrm{K}$ in $10 \mathrm{mM}$ EDTA and $40 \mathrm{mM}$ Tris $\mathrm{pH} 8$ at $45^{\circ} \mathrm{C}$ for $1 \mathrm{~h}$ followed by phenol/chloroform/isoamyl alcohol extraction and ethanol precipitation. The pellet was washed with $70 \% \mathrm{EtOH}$ and resuspended in $50 \mu \mathrm{L}$ molecular grade water. Libraries were prepared using the NuGEN Ovation Ultra Low System V2 kit, according to the manufacturer's instructions, and were sequenced on an Illumina HiSeq 4000 instrument.

ChIP-seq analysis. ChIP-seq fastq reads were aligned to the TAIR10 reference genome with Bowtie using default settings and allowing only uniquely mapping reads. Duplicated reads were removed using SAMtools. IGB genome browser was used to visualize the data and to generate snapshots. Normalized read coverage tracks were generated using the USeq package Sam2Useq application. ChIP-seq peaks in wild type and mutants were called by callpeak function in MACS2 (v2.1.1.). ChIP-seq data metaplots and $k$-means clustering were generated by NGSPLOT (v4.02.48). Enriched motifs in peak sets were identified using HOMER findMotifsGenome. Annotation of peak locations were carried out using the HOMER annotatepeaks function. GO term enrichment was determined using AgriGO.

ATAC-seq. Inflorescence tissues were collected for nuclei extraction as follows. About $5 \mathrm{~g}$ of inflorescence tissue was collected and immediately transferred into ice-cold grinding buffer ( $300 \mathrm{mM}$ sucrose, $20 \mathrm{mM}$ Tris $\mathrm{pH} 8,5 \mathrm{mM} \mathrm{MgCl}_{2}, 5 \mathrm{mM}$ $\mathrm{KCl}, 0.2 \%$ Triton X-100, $5 \mathrm{mM} \beta$-mercaptoethanol, $35 \%$ glycerol). The samples were ground with an Omni International General Laboratory Homogenizer at $4{ }^{\circ} \mathrm{C}$ and then filtered through a two-layer Miracloth and a $40-\mu \mathrm{m}$ nylon mesh Cell Strainer (Fisher). Samples were spin filtered for $10 \mathrm{~min}$ at $3000 \mathrm{~g}$, the supernatant discarded, and the pellet resuspended with $25 \mathrm{~mL}$ of grinding buffer using a Dounce homogenizer. The wash step was repeated once and the nuclei resuspended in $0.5 \mathrm{~mL}$ of freezing buffer ( $50 \mathrm{mM}$ Tris pH $8,5 \mathrm{mM} \mathrm{MgCl}_{2}, 20 \%$ glycerol, $5 \mathrm{mM} \beta$-mercaptoethanol). Nuclei were subjected to transposition reaction with Tn5 (Illumina, cat. no. FC-121-1030) ) $^{73}$. For transposition reaction, $25 \mu \mathrm{L}$ of $2 \times$ DMF (66 mM Tris-acetate ( $\mathrm{pH}=7.8$ ), $132 \mathrm{~K}$-Acetate, $20 \mathrm{mM} \mathrm{Mg-Acetate,} 32 \%$ DMF) was mixed with $2.5 \mu \mathrm{L}$ Tn5 and $22.5 \mu \mathrm{L}$ nuclei suspension and incubated at $37^{\circ} \mathrm{C}$ for $30 \mathrm{~min}^{73}$. Transposed DNA fragments were purified with ChIP DNA Clean \& Concentrator Kit (Zymo, cat. no. D5205) ${ }^{73}$. Libraries were generated using Phusion High-Fidelity DNA Polymerase (NEB, cat. no. M0530S) as follows, $12.5 \mu \mathrm{L}$
$2 \times$ Phusion, $1.25 \mu \mathrm{L} 10 \mathrm{mM}$ Ad1 primer, $1.25 \mu \mathrm{L} 10 \mathrm{mM}$ Ad2 primer, $4 \mu \mathrm{L}$ ddH2O and $6 \mu \mathrm{L}$ purified transposed DNA fragments were mixed and subjected to 12 cycles of PCR amplification $\left(72{ }^{\circ} \mathrm{C}\right.$ for $5 \mathrm{~min}, 12$ cycles of $98^{\circ} \mathrm{C}$ for $3 \mathrm{~min}, 98^{\circ} \mathrm{C}$ for $45 \mathrm{~s}, 63^{\circ} \mathrm{C}$ for $30 \mathrm{~s}$, and $72^{\circ} \mathrm{C}$ for $\left.1 \mathrm{~min}\right)^{73}$. Libraries were sequenced on an Illumina HiSeq 2000 instrument. ATAC-seq read adaptors were removed with trim_galore and then mapped to the Arabidopsis thaliana reference genome TAIR10 using Bowtie (-X $2000-\mathrm{m}$ 1). Chloroplast and mitochondrial DNA aligned reads were filtered out and duplicate reads were removed using SAMtools.

Reporting Summary. Further information on research design is available in the Nature Research Reporting Summary linked to this article.

\section{Data availability}

Data supporting the findings of this work are available within the paper and its Supplementary Information files. A reporting summary for this Article is available as a Supplementary Information file. The datasets generated and analyzed during the current study are available from the corresponding author upon request. The source data underlying Figs. 1a, 1c, 1d, 2a, 2c, 3, and 4, Supplementary Figs. 1, 2, and 6, and Tables 1, 2 are provided as a Source Data file. All high-throughput sequencing data generated in this study is accessible at NCBI's Gene Expression Omnibus (GEO) via GEO Series accession number GSE123263.

Received: 5 December 2018 Accepted: 5 July 2019

Published online: 26 July 2019

\section{References}

1. Luger, K., Rechsteiner, T. J., Flaus, A. J., Waye, M. M. \& Richmond, T. J. Characterization of nucleosome core particles containing histone proteins made in bacteria. J. Mol. Biol. 272, 301-311 (1997).

2. Clapier, C. R., Iwasa, J., Cairns, B. R. \& Peterson, C. L. Mechanisms of action and regulation of ATP-dependent chromatin-remodelling complexes. Nat. Rev. Mol. Cell Biol. 18, 407-422 (2017).

3. Clapier, C. R. \& Cairns, B. R. The biology of chromatin remodeling complexes. Annu. Rev. Biochem. 78, 273-304 (2009).

4. van Daal, A., White, E. M., Elgin, S. C. \& Gorovsky, M. A. Conservation of intron position indicates separation of major and variant H2As is an early event in the evolution of eukaryotes. J. Mol. Evol. 30, 449-455 (1990).

5. Thatcher, T. H. \& Gorovsky, M. A. Phylogenetic analysis of the core histones H2A, H2B, H3, and H4. Nucleic Acids Res. 22, 174-179 (1994).

6. Talbert, P. B. \& Henikoff, S. Histone variants-ancient wrap artists of the epigenome. Nat. Rev. Mol. Cell Biol. 11, 264-275 (2010).

7. Zilberman, D., Coleman-Derr, D., Ballinger, T. \& Henikoff, S. Histone H2A.Z and DNA methylation are mutually antagonistic chromatin marks. Nature 456, 125-129 (2008).

8. Raisner, R. M. et al. Histone variant H2A.Z marks the 5' ends of both active and inactive genes in euchromatin. Cell 123, 233-248 (2005).

9. Zhang, H., Roberts, D. N. \& Cairns, B. R. Genome-wide dynamics of Htz1, a histone $\mathrm{H} 2 \mathrm{~A}$ variant that poises repressed/basal promoters for activation through histone loss. Cell 123, 219-231 (2005).

10. Mavrich, T. N. et al. Nucleosome organization in the Drosophila genome. Nature 453, 358-362 (2008).

11. Millar, C. B., Xu, F., Zhang, K. \& Grunstein, M. Acetylation of H2AZ Lys 14 is associated with genome-wide gene activity in yeast. Genes Dev. 20, 711-722 (2006).

12. Barski, A. et al. High-resolution profiling of histone methylations in the human genome. Cell 129, 823-837 (2007).

13. Hardy, S. et al. The euchromatic and heterochromatic landscapes are shaped by antagonizing effects of transcription on H2A.Z deposition. PLoS Genet. $\mathbf{5}$, e1000687 (2009).

14. Guillemette, B. et al. Variant histone H2A.Z is globally localized to the promoters of inactive yeast genes and regulates nucleosome positioning. PLoS Biol. 3, e384 (2005).

15. Hu, G. et al. H2A.Z facilitates access of active and repressive complexes to chromatin in embryonic stem cell self-renewal and differentiation. Cell Stem Cell 12, 180-192 (2013).

16. Murphy, P. J., Wu, S. F., James, C. R., Wike, C. L. \& Cairns, B. R. Placeholder nucleosomes underlie germline-to-embryo DNA methylation reprogramming. Cell 172, 993-1006, e1013 (2018)

17. Coleman-Derr, D. \& Zilberman, D. Deposition of histone variant H2A.Z within gene bodies regulates responsive genes. PLoS Genet. 8, e1002988 (2012).

18. Bewick, A. J. et al. On the origin and evolutionary consequences of gene body DNA methylation. Proc. Natl Acad. Sci. USA 113, 9111-9116 (2016).

19. Zemach, A., McDaniel, I. E., Silva, P. \& Zilberman, D. Genome-wide evolutionary analysis of eukaryotic DNA methylation. Science 328, 916-919 (2010). 
20. Edwards, J. R. et al. Chromatin and sequence features that define the fine and gross structure of genomic methylation patterns. Genome Res. 20, 972-980 (2010).

21. Conerly, M. L. et al. Changes in H2A.Z occupancy and DNA methylation during B-cell lymphomagenesis. Genome Res. 20, 1383-1390 (2010).

22. Luo, C. \& Lam, E. ANCORP: a high-resolution approach that generates distinct chromatin state models from multiple genome-wide datasets. Plant J. 63, 339-351 (2010).

23. Dai, X. et al. H2A.Z represses gene expression by modulating promoter nucleosome structure and enhancer histone modifications in Arabidopsis. Mol. Plant 10, 1274-1292 (2017).

24. Sura, W. et al. Dual role of the histone variant H2A.Z in transcriptional regulation of stress-response genes. Plant Cell 29, 791-807 (2017).

25. Kumar, S. V. \& Wigge, P. A. H2A.Z-containing nucleosomes mediate the thermosensory response in Arabidopsis. Cell 140, 136-147 (2010).

26. Carter, B. et al. The chromatin remodelers PKL and PIE1 act in an epigenetic pathway that determines H3K27me3 homeostasis in Arabidopsis. Plant Cell 30, 1337-1352 (2018).

27. Mizuguchi, G. et al. ATP-driven exchange of histone $\mathrm{H} 2 \mathrm{AZ}$ variant catalyzed by SWR1 chromatin remodeling complex. Science 303, 343-348 (2004).

28. Krogan, N. J. et al. Regulation of chromosome stability by the histone H2A variant Htz1, the Swr1 chromatin remodeling complex, and the histone acetyltransferase NuA4. Proc. Natl Acad. Sci. USA 101, 13513-13518 (2004).

29. Kobor, M. S. et al. A protein complex containing the conserved Swi2/Snf2related ATPase Swrlp deposits histone variant H2A.Z into euchromatin. PLoS Biol. 2, E131 (2004).

30. Nguyen, V. Q. et al. Molecular architecture of the ATP-dependent chromatinremodeling complex SWR1. Cell 154, 1220-1231 (2013).

31. Cai, Y. et al. The mammalian YL1 protein is a shared subunit of the TRRAP/ TIP60 histone acetyltransferase and SRCAP complexes. J. Biol. Chem. 280, 13665-13670 (2005).

32. Ruhl, D. D. et al. Purification of a human SRCAP complex that remodels chromatin by incorporating the histone variant H2A.Z into nucleosomes. Biochemistry 45, 5671-5677 (2006).

33. Wong, M. M., Cox, L. K. \& Chrivia, J. C. The chromatin remodeling protein, SRCAP, is critical for deposition of the histone variant H2A.Z at promoters. J. Biol. Chem. 282, 26132-26139 (2007).

34. Verbsky, M. L. \& Richards, E. J. Chromatin remodeling in plants. Curr. Opin. Plant Biol. 4, 494-500 (2001).

35. Choi, K. et al. Arabidopsis homologs of components of the SWR1 complex regulate flowering and plant development. Development 134, 1931-1941 (2007).

36. Lazaro, A., Gomez-Zambrano, A., Lopez-Gonzalez, L., Pineiro, M. \& Jarillo, J. A. Mutations in the Arabidopsis SWC6 gene, encoding a component of the SWR1 chromatin remodelling complex, accelerate flowering time and alter leaf and flower development. J. Exp. Bot. 59, 653-666 (2008).

37. March-Diaz, R., Garcia-Dominguez, M., Florencio, F. J. \& Reyes, J. C. SEF, a new protein required for flowering repression in Arabidopsis, interacts with PIE1 and ARP6. Plant Physiol. 143, 893-901 (2007).

38. Gomez-Zambrano, A. et al. Arabidopsis SWC4 binds DNA and recruits the SWR1 complex to modulate histone H2A.Z deposition at key regulatory genes. Mol. Plant 11, 815-832 (2018).

39. Choi, K. et al. SUPPRESSOR OF FRIGIDA3 encodes a nuclear ACTINRELATED PROTEIN6 required for floral repression in Arabidopsis. Plant Cell 17, 2647-2660 (2005).

40. Deal, R. B., Kandasamy, M. K., McKinney, E. C. \& Meagher, R. B. The nuclear actin-related protein ARP6 is a pleiotropic developmental regulator required for the maintenance of FLOWERING LOCUS C expression and repression of flowering in Arabidopsis. Plant Cell 17, 2633-2646 (2005).

41. Martin-Trillo, M. et al. EARLY IN SHORT DAYS 1 (ESD1) encodes ACTINRELATED PROTEIN 6 (AtARP6), a putative component of chromatin remodelling complexes that positively regulates FLC accumulation in Arabidopsis. Development 133, 1241-1252 (2006).

42. Ranjan, A. et al. Nucleosome-free region dominates histone acetylation in targeting SWR1 to promoters for H2A.Z replacement. Cell 154, 1232-1245 (2013).

43. Yen, K., Vinayachandran, V. \& Pugh, B. F. SWR-C and INO80 chromatin remodelers recognize nucleosome-free regions near +1 nucleosomes. Cell 154, 1246-1256 (2013).

44. Altaf, M. et al. NuA4-dependent acetylation of nucleosomal histones $\mathrm{H} 4$ and $\mathrm{H} 2 \mathrm{~A}$ directly stimulates incorporation of H2A.Z by the SWR1 complex. J. Biol. Chem. 285, 15966-15977 (2010).

45. Babiarz, J. E., Halley, J. E. \& Rine, J. Telomeric heterochromatin boundaries require NuA4-dependent acetylation of histone variant $\mathrm{H} 2 \mathrm{~A} . \mathrm{Z}$ in Saccharomyces cerevisiae. Genes Dev. 20, 700-710 (2006).

46. Durant, M. \& Pugh, B. F. NuA4-directed chromatin transactions throughout the Saccharomyces cerevisiae genome. Mol. Cell Biol. 27, 5327-5335 (2007).

47. Galarneau, L. et al. Multiple links between the NuA4 histone acetyltransferase complex and epigenetic control of transcription. Mol. Cell 5, 927-937 (2000).
48. Le Masson, I. et al. Yaf9, a novel NuA4 histone acetyltransferase subunit, is required for the cellular response to spindle stress in yeast. Mol. Cell Biol. 23 6086-6102 (2003)

49. Zhang, H. et al. The Yaf9 component of the SWR1 and NuA4 complexes is required for proper gene expression, histone $\mathrm{H} 4$ acetylation, and Htzl replacement near telomeres. Mol. Cell Biol. 24, 9424-9436 (2004).

50. Lu, P. Y., Levesque, N. \& Kobor, M. S. NuA4 and SWR1-C: two chromatinmodifying complexes with overlapping functions and components. Biochem Cell Biol. 87, 799-815 (2009).

51. Choi, J., Heo, K. \& An, W. Cooperative action of TIP48 and TIP49 in H2A.Z exchange catalyzed by acetylation of nucleosomal H2A. Nucleic Acids Res 37, 5993-6007 (2009).

52. Jarillo, J. A. \& Pineiro, M. H2A.Z mediates different aspects of chromatin function and modulates flowering responses in Arabidopsis. Plant J. 83, 96-109 (2015).

53. Crevillen, P. et al. Arabidopsis YAF9 histone readers modulate flowering time through NuA4-complex-dependent $\mathrm{H} 4$ and H2A.Z histone acetylation at FLC chromatin. New Phytol. 222, 1893-1908 (2019).

54. Sijacic, P., Holder, D. H., Bajic, M. \& Deal, R. B.. Methyl-CpG-binding domain 9 (MBD9) is required for H2A.Z incorporation into chromatin at a subset of H2A.Z-enriched regions in the Arabidopsis genome. Preprint at https://www. biorxiv.org/content/10.1101/404152v1 (2018).

55. Peng, M., Cui, Y., Bi, Y. M. \& Rothstein, S. J. AtMBD9: a protein with a methyl-CpG-binding domain regulates flowering time and shoot branching in Arabidopsis. Plant J. 46, 282-296 (2006).

56. Yaish, M. W., Peng, M. \& Rothstein, S. J. AtMBD9 modulates Arabidopsis development through the dual epigenetic pathways of DNA methylation and histone acetylation. Plant J. 59, 123-135 (2009).

57. Wei, W. et al. The Alfin-like homeodomain finger protein AL5 suppresses multiple negative factors to confer abiotic stress tolerance in Arabidopsis. Plant J. 81, 871-883 (2015).

58. Deal, R. B., Topp, C. N., McKinney, E. C. \& Meagher, R. B. Repression of flowering in Arabidopsis requires activation of FLOWERING LOCUS C expression by the histone variant H2A.Z. Plant Cell 19, 74-83 (2007).

59. March-Diaz, R. et al. Histone H2A.Z and homologues of components of the SWR1 complex are required to control immunity in Arabidopsis. Plant J. 53, 475-487 (2008).

60. Smith, A. P. et al. Histone H2A.Z regulates the expression of several classes of phosphate starvation response genes but not as a transcriptional activator. Plant Physiol. 152, 217-225 (2010).

61. Berg, A. et al. Ten members of the Arabidopsis gene family encoding methyl CpG-binding domain proteins are transcriptionally active and at least one, AtMBD11, is crucial for normal development. Nucleic Acids Res. 31 , 5291-5304 (2003)

62. Greenberg, M. V. et al. Interplay between active chromatin marks and RNAdirected DNA methylation in Arabidopsis thaliana. PLoS Genet. 9, e1003946 (2013).

63. Li, C. et al. The Arabidopsis SWI2/SNF2 chromatin Remodeler BRAHMA regulates polycomb function during vegetative development and directly activates the flowering repressor gene SVP. PLoS Genet. 11, e1004944 (2015).

64. Zhong, X. et al. DDR complex facilitates global association of RNA polymerase V to promoters and evolutionarily young transposons. Nat. Struct. Mol. Biol. 19, 870-875 (2012).

65. Brzezinka, K. et al. Arabidopsis FORGETTER1 mediates stress-induced chromatin memory through nucleosome remodeling. Elife 5, e17061 (2016).

66. Smaczniak, C. et al. Characterization of MADS-domain transcription factor complexes in Arabidopsis flower development. Proc. Natl Acad. Sci. USA 109, 1560-1565 (2012).

67. Dong, J. et al. SLIDE, the protein interacting domain of Imitation Switch remodelers, binds DDT-domain proteins of different subfamilies in chromatin remodeling complexes. J. Integr. Plant Biol. 55, 928-937 (2013).

68. Santisteban, M. S., Kalashnikova, T. \& Smith, M. M. Histone H2A.Z regulats transcription and is partially redundant with nucleosome remodeling complexes. Cell 103, 411-422 (2000).

69. $\mathrm{Li}, \mathrm{G}$. et al. Imitation Switch chromatin remodeling factors and their interacting RINGLET proteins act together in controlling the plant vegetative phase in Arabidopsis. Plant J. 72, 261-270 (2012).

70. $\mathrm{Li}, \mathrm{G}$. et al. ISWI proteins participate in the genome-wide nucleosome distribution in Arabidopsis. Plant J. 78, 706-714 (2014).

71. Bieluszewski, T. et al. AtEAF1 is a potential platform protein for Arabidopsis NuA4 acetyltransferase complex. BMC Plant Biol. 15, 75 (2015).

72. Pfab, A., Bruckmann, A., Nazet, J., Merkl, R. \& Grasser, K. D. The Adaptor protein ENY2 is a component of the deubiquitination module of the Arabidopsis SAGA transcriptional co-activator complex but not of the TREX2 complex. J. Mol. Biol. 430, 1479-1494 (2018).

73. Buenrostro, J. D., Giresi, P. G., Zaba, L. C., Chang, H. Y. \& Greenleaf, W. J. Transposition of native chromatin for fast and sensitive epigenomic profiling 
of open chromatin, DNA-binding proteins and nucleosome position. Nat. Methods 10, 1213-1218 (2013).

\section{Acknowledgements}

We thank Mahnaz Akhavan for support with high-throughput sequencing at the University of California, Los Angeles (UCLA) Broad Stem Cell Research Center BioSequencing Core Facility. Work in the Jacobsen lab was supported by NIH grant R35 GM130272. M.E.P. was supported by the Damon Runyon Cancer Foundation DRG:2217-15. Work in the I.A. laboratory was supported by the National Science Foundation of China (grant numbers: 31571328 and 31870270). S.E.J. is an Investigator of the Howard Hughes Medical Institute.

\section{Author contributions}

M.E.P. performed RNA-seq and ChIP-seq and generated sequencing libraries. Y.W. and L.X. developed ARP6-tagged lines and performed IP, co-IP, western blots, RT-PCRs, and genetic analyses. M.E.P. and B.N. developed MBD9-tagged lines. M.E.P., Z.Z. and W.L. performed data bioinformatics analysis. Z.Z. and W.L. performed ATAC-seq. S.F. performed high-throughput sequencing. S.R. and J.A.W. performed mass spectrometry. M.E.P., I.A., and S.E.J. wrote the manuscript. M.E.P., Z.W., I.A. and S.E.J. coordinated the research.

\section{Additional information}

Supplementary Information accompanies this paper at https://doi.org/10.1038/s41467019-11291-w.
Competing interests: The authors declare no competing interests.

Reprints and permission information is available online at http://npg.nature.com/ reprintsandpermissions/

Peer review information: Nature Communications thanks the anonymous reviewers for their contribution to the peer review of this work.

Publisher's note: Springer Nature remains neutral with regard to jurisdictional claims in published maps and institutional affiliations.

(c) (i) Open Access This article is licensed under a Creative Commons Attribution 4.0 International License, which permits use, sharing, adaptation, distribution and reproduction in any medium or format, as long as you give appropriate credit to the original author(s) and the source, provide a link to the Creative Commons license, and indicate if changes were made. The images or other third party material in this article are included in the article's Creative Commons license, unless indicated otherwise in a credit line to the material. If material is not included in the article's Creative Commons license and your intended use is not permitted by statutory regulation or exceeds the permitted use, you will need to obtain permission directly from the copyright holder. To view a copy of this license, visit http://creativecommons.org/ licenses/by/4.0/.

(C) The Author(s) 2019 